\title{
The TRENDS High-contrast Imaging Survey. VIII. Compendium of Benchmark Objects
}

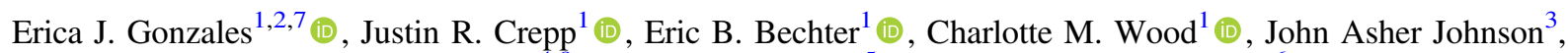 \\ Benjamin T. Montet ${ }^{4,8}$ (D), Howard Isaacson ${ }^{5}$ (ID), and Andrew W. Howard ${ }^{6}$ (D) \\ ${ }^{1}$ Department of Physics, University of Notre Dame, 225 Nieuwland Science Hall, Notre Dame, IN 46556, USA; erjagonz@ucsc.edu \\ ${ }^{2}$ University of California, Santa Cruz, 1156 High Street, Santa Cruz, CA 95065, USA \\ ${ }^{3}$ Harvard Center for Astrophysics, 60 Garden Street, Cambridge, MA 02138, USA \\ ${ }_{5}^{4}$ School of Physics, University of New South Wales, Sydney, NSW 2052, Australia \\ ${ }^{5}$ University of California, Berkeley, 501 Campbell Hall, Berkeley, CA 94720, USA \\ ${ }^{6}$ California Institute of Technology, 1200 E. California Blvd., Pasadena, CA 91125, USA \\ Received 2019 February 13; revised 2020 January 15; accepted 2020 January 17; published 2020 April 9
}

\begin{abstract}
The physical properties of faint stellar and substellar objects often rely on indirect, model-dependent estimates. For example, the masses of brown dwarfs are usually inferred using evolutionary models, which are age dependent and have yet to be properly calibrated. With the goal of identifying new benchmark objects to test low-mass stellar and substellar models, we have carried out a comprehensive adaptive optics survey as part of the TaRgetting bENchmark-objects with the Doppler Spectroscopy high-contrast imaging program. Using legacy radial velocity measurements from the High Resolution Echelle Spectrometer at Keck, we have identified several dozen stars that show long-term Doppler accelerations. We present follow-up high-contrast observations from the campaign and report the discovery of 31 comoving companions, as well as 11 strong candidate companions, to solar-type stars with well-determined parallax and metallicity values. Benchmark objects of this nature lend themselves to orbit determinations, dynamical mass estimates, and independent compositional assessment. This compendium of benchmark objects will serve as a convenient test group to substantiate theoretical evolutionary and atmospheric models near the hydrogen fusing limit.
\end{abstract}

Unified Astronomy Thesaurus concepts: Astrometric binary stars (79); Binary stars (154); Visual binary stars (1777); Spectroscopic binary stars (1557); Direct imaging (387); Brown dwarfs (185); Low mass stars (2050); Radial velocity (1332); M stars (985)

Supporting material: machine-readable tables

\section{Introduction}

Very low mass (VLM) stars and brown dwarfs (BDs) represent some of the most abundant objects in the universe. Studying their physical properties has implications for our understanding of star formation and evolution, as well as the detection and characterization of extrasolar planets (Lada \& Lada 2003; Muirhead et al. 2018). Mass is of particular interest as it fundamentally governs the evolution and fate of stellar and substellar objects. Comprising the spectral classes M, L, T, and $\mathrm{Y}$, VLM stars and BDs span $\approx 1.5$ dex of dynamic range in mass. Accurate mass estimates, however, are difficult to obtain due to their cool, dense nature (Liu et al. 2002; Kirkpatrick et al. 2012; Dupuy \& Liu 2017). Instead, theoretical models are used to infer physical parameters.

Mass, radius, age, and composition often represent degenerate model variables. As such, stellar and substellar "benchmark" systems with directly measurable physical properties serve as convenient laboratories for testing theoretical models (Chabrier et al. 2000; Allard et al. 2001, 2013; Burrows 2001; Liu et al. 2008; Saumon \& Marley 2008; Boyajian et al. 2012; Konopacky 2013; Baraffe et al. 2015; Biller et al. 2015; Dupuy et al. 2015; Mann et al. 2017; Crepp et al. 2016, 2018; Dupuy \& Liu 2017; Bowler et al. 2018). Dynamical masses of VLM stars and BDs can be determined using laser guide star adaptive optics (AO) to spatially resolve the individual components of tight binary and multi-star systems (Liu et al. 2008). Likewise,

\footnotetext{
7 National Science Foundation Graduate Research Fellow.

8 Sagan Fellow.
}

faint companions to nearby main-sequence stars can be detected with high-contrast imaging; such objects are particularly valuable as they allow for distance, age, and composition to be inferred from the well-characterized parent star (Liu et al. 2008; Crepp et al. 2014, 2016; Bowler et al. 2018; Cheetham et al. 2018; Wood et al. 2019).

The TaRgetting bENchmark-objects with Doppler Spectroscopy (TRENDS) high-contrast imaging survey uses AO imaging and coronagraphy to search for companions in the VLM star, BD, and exoplanet regime (Crepp et al. 2012b, 2013a, 2013b). In this paper, we report the discovery of 31 comoving companions, as well as 11 strong candidate companions, to nearby solar-type stars. Of these, six companions are found to be members of triple star systems. Targets were selected from the California Planet Search (CPS) program (Howard et al. 2010). In addition to exhibiting a long-term radial velocity (RV) acceleration ("trend"), which was used to identify previously unseen candidate companions, each star also has a welldetermined parallax and metallicity (Tables 1,2 ).

The Doppler RV method benefits from the longest time baselines of any exoplanet detection method. Beyond the orbital radii of planets with already well-characterized Keplerian motions, many stars exhibit accelerations for which only partial orbits or systemic trends in their time series measurements can be discerned (Crepp et al. 2012a). Largely unpublished to date, these data sets lend themselves to early studies of more distant, and necessarily more massive, objects that reside in the outer extremities of other solar systems at projected separations accessible to direct imaging. The 
Table 1

TRENDS System Parameters

\begin{tabular}{|c|c|c|c|c|c|}
\hline$\overline{\text { Star }}$ & R.A. & Decl. & $\pi$ (mas) & $\mu_{\alpha}\left({\left.\text { mas } \mathrm{yr}^{-1}\right)}^{-1}\right.$ & $\mu_{\delta}\left(\right.$ mas yr $\left.^{-1}\right)$ \\
\hline HD 224983 & 000221.54 & +110022.46 & $29.52 \pm 0.06$ & $-4.95 \pm 0.11$ & $-42.71 \pm 0.07$ \\
\hline HIP 1294 & 001611.59 & +303157.28 & $25.46 \pm 0.12$ & $189.89 \pm 0.17$ & $47.84 \pm 0.16$ \\
\hline HD 1205 & 001623.46 & -223516.72 & $15.57 \pm 0.80$ & $19.96 \pm 1.43$ & $-42.68 \pm 1.60$ \\
\hline HD 1384 & 001812.14 & +522445.23 & $6.24 \pm 0.14$ & $-17.50 \pm 0.20$ & $-53.50 \pm 0.20$ \\
\hline HD 6512 & 010612.63 & +131509.88 & $18.36 \pm 0.06$ & $166.03 \pm 0.10$ & $-10.86 \pm 0.07$ \\
\hline HD 31018 & 045322.05 & +241038.47 & $9.93 \pm 0.06$ & $21.14 \pm 0.10$ & $-17.74 \pm 0.06$ \\
\hline HD 34721 & 051850.47 & -180748.19 & $40.47 \pm 0.06$ & $386.01 \pm 0.10$ & $61.01 \pm 0.10$ \\
\hline HD 40647 & 060605.77 & +692834.42 & $31.86 \pm 0.16$ & $-165.11 \pm 0.32$ & $-82.47 \pm 0.32$ \\
\hline HD 50639 & 065344.83 & -093055.67 & $25.68 \pm 0.06$ & $-69.04 \pm 0.08$ & $-31.34 \pm 0.07$ \\
\hline HD 85472 & 095357.96 & +574101.33 & $13.16 \pm 0.03$ & $36.51 \pm 0.05$ & $-92.28 \pm 0.05$ \\
\hline HD 88986 & 101628.08 & +284056.94 & $30.03 \pm 0.04$ & $-65.69 \pm 0.09$ & $-95.51 \pm 0.07$ \\
\hline HIP 55507 & 112205.75 & +465430.18 & $39.20 \pm 0.04$ & $-197.56 \pm 0.04$ & $-134.88 \pm 0.04$ \\
\hline HD 110537 & 124259.33 & -040257.60 & $22.15 \pm 0.05$ & $-219.37 \pm 0.11$ & $-179.68 \pm 0.06$ \\
\hline HD 111031 & 124630.84 & -114844.79 & $32.02 \pm 0.05$ & $-279.79 \pm 0.09$ & $46.71 \pm 0.07$ \\
\hline HIP 63762 & 130407.28 & +870655.55 & $39.51 \pm 0.04$ & $-92.16 \pm 0.10$ & $181.12 \pm 0.07$ \\
\hline HD 129191 & 144116.18 & -045641.53 & $18.39 \pm 0.05$ & $26.42 \pm 0.09$ & $-152.97 \pm 0.08$ \\
\hline HD 129814 & 144411.69 & +182743.62 & $23.81 \pm 0.06$ & $-59.34 \pm 0.07$ & $-165.08 \pm 0.09$ \\
\hline HD 136274 & 151859.05 & +254130.29 & $29.42 \pm 0.05$ & $-564.57 \pm 0.06$ & $-125.14 \pm 0.07$ \\
\hline HD 139457 & 153759.21 & +101423.56 & $20.82 \pm 0.05$ & $129.82 \pm 0.08$ & $-358.77 \pm 0.08$ \\
\hline HD 142229 & 155320.01 & +041511.70 & $21.62 \pm 0.04$ & $-34.24 \pm 0.08$ & $9.29 \pm 0.06$ \\
\hline HD 147231 & 161450.25 & +705546.80 & $24.86 \pm 0.02$ & $-5.28 \pm 0.05$ & $-287.27 \pm 0.05$ \\
\hline HD 155413 & 171158.64 & -143713.37 & $11.85 \pm 0.05$ & $-18.50 \pm 0.09$ & $-39.82 \pm 0.07$ \\
\hline HD 157338 & 172408.74 & -344754.44 & $30.18 \pm 0.05$ & $-0.39 \pm 0.72$ & $-182.73 \pm 0.37$ \\
\hline HD 164509 & 180131.23 & +000616.40 & $18.80 \pm 0.05$ & $-7.86 \pm 0.09$ & $-20.38 \pm 0.09$ \\
\hline HD 180684 & 191648.59 & +185834.74 & $17.85 \pm 0.04$ & $10.68 \pm 0.05$ & $-65.08 \pm 0.06$ \\
\hline HD 183473 & 192757.24 & +424635.90 & $11.16 \pm 0.03$ & $68.15 \pm 0.05$ & $160.24 \pm 0.05$ \\
\hline HD 196201 & 203526.23 & +112125.66 & $24.32 \pm 0.11$ & $72.52 \pm 0.15$ & $366.03 \pm 0.11$ \\
\hline HD 201924 & $2111 \quad 10.85$ & +452721.29 & $31.85 \pm 0.04$ & $-239.39 \pm 0.06$ & $-300.78 \pm 0.06$ \\
\hline HD 213519 & 223155.72 & +450842.35 & $24.36 \pm 0.03$ & $-174.73 \pm 0.05$ & $34.55 \pm 0.05$ \\
\hline HD 1293 & 001705.55 & -013910.85 & $5.13 \pm 0.19$ & $20.10 \pm 0.44$ & $10.41 \pm 0.24$ \\
\hline HD 1388 & 001758.87 & -132720.31 & $37.11 \pm 0.05$ & $401.22 \pm 0.13$ & $-0.13 \pm 0.05$ \\
\hline HD 4406 & 004651.74 & +462148.99 & $8.03 \pm 0.09$ & $-24.54 \pm 0.10$ & $-21.47 \pm 0.16$ \\
\hline HD 6558 & 010625.72 & -004458.03 & $12.18 \pm 0.06$ & $70.80 \pm 0.10$ & $-35.26 \pm 0.06$ \\
\hline HIP 46199 & 092510.78 & +460553.65 & $44.40 \pm 0.82$ & $-221.04 \pm 1.20$ & $24.98 \pm 1.20$ \\
\hline HD 103829 & 115731.21 & +533315.09 & $11.25 \pm 1.37$ & $44.02 \pm 0.98$ & $-10.98 \pm 0.97$ \\
\hline HD 105618 & 120936.62 & +111241.68 & $14.24 \pm 0.05$ & $-104.61 \pm 0.10$ & $-1.96 \pm 0.05$ \\
\hline HD 131509 & 145311.92 & +283029.78 & $12.91 \pm 0.05$ & $-94.09 \pm 0.08$ & $86.55 \pm 0.10$ \\
\hline HD 156826 & 171959.55 & -055503.02 & $20.94 \pm 0.04$ & $45.29 \pm 0.08$ & $-193.00 \pm 0.06$ \\
\hline HD 217165 & 225829.88 & +094932.01 & $22.74 \pm 0.05$ & $109.66 \pm 0.10$ & $-1.44 \pm 0.07$ \\
\hline
\end{tabular}

Note. Right ascension (R.A.), declination (decl.), parallax $(\pi)$, and proper motions $\left(\mu_{\alpha}, \mu_{\delta}\right)$ are taken from the Gaia Data Release 2 (Gaia Collaboration et al. 2018). The horizontal line separates systems with confirmed companions (above) and systems with candidate companions (below).

combination of methods, precision RVs with high-resolution AO imaging, produces a more holistic view of stellar and extrasolar planet orbital architectures by allowing for further inferences of formation, dynamical hierarchies, interactions, and their evolution.

We present RV time series data, AO imaging observations, and multi-epoch astrometry measurements for the companions detected. A number of the detected companions will lend themselves to precise dynamical mass estimates with continued $\mathrm{RV}$ and AO imaging follow-up observations.

\section{Target Selection}

Targets were selected by analyzing legacy RV time series data obtained from the High Resolution Echelle Spectrometer (HIRES) at Keck (Marcy \& Butler 1992; Vogt et al. 1994; Howard et al. 2010). Complementary RV data was also obtained from the publicly available Lick-Carnegie Exoplanet
Survey (Butler et al. 2017). Visual inspection of the RV time series was performed and, if need be, analyzed using a statistical Bayesian Inference Criteria to compare marginal accelerations with the null hypothesis of a straight line of zero slope. Stars exhibiting long-term RV accelerations were (re-) observed based in part on the amount of time baseline that could be gained from an additional measurement. In the case where systems showed marginal trends, several measurements were obtained to improve time sampling and the signal-to-noise ratio of the acceleration. Those with fewer than $N \approx 10$ measurements over a several year time span were generally included as lower-priority targets for planning purposes depending on the logistics of a given observing run. Those confirmed as comoving using multi-epoch TRENDS imaging observations now have a median time baseline of $10.41 \mathrm{yr}$ from precision stellar $\mathrm{RVs}$, and $2.2 \mathrm{yr}$ from our direct imaging astrometric follow up. A table of observations and observational set-up is listed in Appendix A.1. 
Table 2

Spectral-type, Metallicity, and Apparent Magnitudes

\begin{tabular}{|c|c|c|c|c|c|c|c|}
\hline Star & SpTy & {$[\mathrm{Fe} / \mathrm{H}]$} & $B$ & V & $J$ & $H$ & $K_{s}$ \\
\hline HD 224983 & $\mathrm{~K} 0 \mathrm{~V}$ & $0.00 \pm 0.03$ & $9.33 \pm 0.01$ & $8.47 \pm 0.01$ & $\ldots$ & $\ldots$ & $\ldots$ \\
\hline HIP 1294 & K4V & $0.17 \pm 0.04$ & $9.84 \pm 0.03$ & $8.82 \pm 0.01$ & $6.92 \pm 0.02$ & $6.40 \pm 0.04$ & $6.31 \pm 0.02$ \\
\hline HD 1205 & G3V & $0.30 \pm 0.04$ & $8.58 \pm 0.02$ & $7.90 \pm 0.01$ & $6.73 \pm 0.02$ & $6.44 \pm 0.02$ & $6.36 \pm 0.02$ \\
\hline HD 1384 & G5 & $0.26 \pm 0.04$ & $9.13 \pm 0.01$ & $8.10 \pm 0.01$ & $6.37 \pm 0.02$ & $5.90 \pm 0.02$ & $5.78 \pm 0.02$ \\
\hline HD 6512 & G0 & $0.12 \pm 0.04$ & $8.81 \pm 0.02$ & $8.15 \pm 0.01$ & $6.89 \pm 0.02$ & $6.64 \pm 0.02$ & $6.57 \pm 0.02$ \\
\hline HD 17230 & K6V & $0.09 \pm 0.04$ & 9.85 & 8.57 & $6.29 \pm 0.02$ & $5.69 \pm 0.03$ & $5.53 \pm 0.02$ \\
\hline HD 31018 & F8 & $0.18 \pm 0.04$ & $8.28 \pm 0.02$ & $7.62 \pm 0.01$ & $6.45 \pm 0.03$ & $6.16 \pm 0.02$ & $6.08 \pm 0.02$ \\
\hline HD 34721 & G0V & $-0.07 \pm 0.03$ & 6.54 & 5.966 & $5.18 \pm 0.27$ & $4.75 \pm 0.27$ & $4.55 \pm 0.02$ \\
\hline HD 40647 & G5V & $-0.06 \pm 0.04$ & $9.06 \pm 0.02$ & $8.26 \pm 0.01$ & $6.81 \pm 0.03$ & $6.43 \pm 0.04$ & $6.33 \pm 0.02$ \\
\hline HD 50639 & $\mathrm{~F} 8.5 \mathrm{~V}$ & $-0.02 \pm 0.03$ & $7.59 \pm 0.02$ & $7.05 \pm 0.01$ & $6.04 \pm 0.03$ & $5.81 \pm 0.03$ & $5.69 \pm 0.02$ \\
\hline HD 85472 & G8IV & $-0.05 \pm 0.04$ & $8.25 \pm 0.01$ & $7.45 \pm 0.01$ & $5.99 \pm 0.02$ & $5.59 \pm 0.03$ & $5.55 \pm 0.06$ \\
\hline HD 88986 & $\mathrm{G} 2 \mathrm{~V}$ & $0.09 \pm 0.03$ & $7.10 \pm 0.02$ & $6.47 \pm 0.01$ & $5.2 \pm 0.02$ & $4.95 \pm 0.02$ & $4.88 \pm 0.02$ \\
\hline HIP 55507 & K6V & $-0.05 \pm 0.04$ & $11.18 \pm 0.07$ & $9.79 \pm 0.03$ & $7.36 \pm 0.02$ & $6.76 \pm 0.02$ & $6.61 \pm 0.02$ \\
\hline HD 110537 & $\mathrm{G} 6 / 8 \mathrm{~V}$ & $0.12 \pm 0.03$ & $8.51 \pm 0.02$ & $7.83 \pm 0.01$ & $6.58 \pm 0.02$ & $6.33 \pm 0.05$ & $6.20 \pm 0.02$ \\
\hline HD 111031 & G5V & $0.28 \pm 0.03$ & 7.57 & 6.87 & $5.74 \pm 0.02$ & $5.41 \pm 0.04$ & $5.32 \pm 0.03$ \\
\hline HIP 63762 & K0 & $0.09 \pm 0.04$ & $9.91 \pm 0.03$ & $8.81 \pm 0.01$ & $6.81 \pm 0.02$ & $6.26 \pm 0.02$ & $6.16 \pm 0.02$ \\
\hline HD 129191 & G6V & $0.24 \pm 0.03$ & $8.87 \pm 0.02$ & $8.21 \pm 0.02$ & $7.04 \pm 0.04$ & $6.74 \pm 0.04$ & $6.68 \pm 0.02$ \\
\hline HD 129814 & G5V & $0.00 \pm 0.03$ & $8.16 \pm 0.02$ & $7.52 \pm 0.01$ & $6.33 \pm 0.02$ & $6.07 \pm 0.02$ & $5.99 \pm 0.02$ \\
\hline HD 136274 & G8V & $-0.23 \pm 0.03$ & 8.70 & 7.96 & $6.53 \pm 0.02$ & $6.21 \pm 0.03$ & $6.12 \pm 0.02$ \\
\hline HD 139457 & F8V & $-0.48 \pm 0.03$ & $7.62 \pm 0.01$ & $7.10 \pm 0.01$ & $6.05 \pm 0.03$ & $5.77 \pm 0.03$ & $5.70 \pm 0.02$ \\
\hline HD 142229 & G5V & $0.05 \pm 0.03$ & $8.70 \pm 0.02$ & $8.08 \pm 0.01$ & $6.97 \pm 0.04$ & $6.67 \pm 0.03$ & $6.61 \pm 0.02$ \\
\hline HD 147231 & G5V & $-0.00 \pm 0.03$ & 8.53 & 7.90 & $6.56 \pm 0.03$ & $6.23 \pm 0.02$ & $6.15 \pm 0.02$ \\
\hline HD 155413 & G3III/IV & $0.26 \pm 0.04$ & $7.94 \pm 0.02$ & $7.26 \pm 0.01$ & $6.03 \pm 0.02$ & $5.73 \pm 0.03$ & $5.63 \pm 0.02$ \\
\hline HD 157338 & F9.5V & $-0.08 \pm 0.03$ & $7.50 \pm 0.01$ & $6.92 \pm 0.01$ & $5.87 \pm 0.03$ & $5.58 \pm 0.04$ & $5.49 \pm 0.03$ \\
\hline HD 164509 & G2V & $0.21 \pm 0.04$ & $8.72 \pm 0.02$ & $8.10 \pm 0.01$ & $6.94 \pm 0.03$ & $6.67 \pm 0.06$ & $6.58 \pm 0.02$ \\
\hline HD 180684 & F8V & $0.05 \pm 0.03$ & $7.57 \pm 0.01$ & $7.02 \pm 0.01$ & $5.98 \pm 0.03$ & $5.74 \pm 0.02$ & $5.71 \pm 0.02$ \\
\hline HD 183473 & G5 & $-0.06 \pm 0.04$ & $8.61 \pm 0.02$ & $7.88 \pm 0.01$ & $6.49 \pm 0.02$ & $6.17 \pm 0.02$ & $6.16 \pm 9.99$ \\
\hline HD 196201 & G5 & $-0.14 \pm 0.03$ & $9.25 \pm 0.02$ & $8.48 \pm 0.01$ & $7.07 \pm 0.03$ & $6.67 \pm 0.04$ & $6.56 \pm 0.02$ \\
\hline HD 201924 & K0 & $0.10 \pm 0.04$ & 8.59 & 7.81 & $6.41 \pm 0.03$ & $6.06 \pm 0.02$ & $5.99 \pm 0.02$ \\
\hline HD 213519 & G5 & $-0.00 \pm 0.03$ & $8.32 \pm 0.01$ & $7.68 \pm 0.01$ & $6.47 \pm 0.02$ & $6.20 \pm 0.02$ & $6.14 \pm 0.02$ \\
\hline HD 1293 & G6V & $-0.38 \pm 0.04$ & $9.14 \pm 0.02$ & $8.37 \pm 0.01$ & $6.88 \pm 0.02$ & $6.42 \pm 0.03$ & $6.35 \pm 0.02$ \\
\hline HD 1388 & G0V & $-0.00 \pm 0.04$ & 7.09 & 6.50 & $5.38 \pm 0.03$ & $5.07 \pm 0.03$ & $4.99 \pm 0.02$ \\
\hline HD 4406 & G5 & $0.41 \pm 0.04$ & $8.20 \pm 0.02$ & $7.48 \pm 0.01$ & $6.20 \pm 0.02$ & $5.93 \pm 0.02$ & $5.82 \pm 0.02$ \\
\hline HD 6558 & G2V & $0.26 \pm 0.03$ & 8.81 & 8.20 & $7.19 \pm 0.02$ & $6.95 \pm 0.03$ & $6.86 \pm 0.02$ \\
\hline HIP 46199 & K4V & $\ldots$ & $10.22 \pm 0.03$ & $9.14 \pm 0.02$ & $6.91 \pm 0.02$ & $6.35 \pm 0.03$ & $6.19 \pm 0.02$ \\
\hline HD 103829 & F8 & $0.12 \pm 0.03$ & $9.91 \pm 0.01$ & $9.24 \pm 0.01$ & $7.97 \pm 0.03$ & $7.67 \pm 0.02$ & $7.57 \pm 0.03$ \\
\hline HD 105618 & G0 & $\ldots$ & 9.30 & 8.64 & $7.42 \pm 0.03$ & $7.14 \pm 0.03$ & $7.01 \pm 0.02$ \\
\hline HD 131509 & $\mathrm{~K} 0 \mathrm{~V}$ & $-0.11 \pm 0.03$ & 8.82 & 7.93 & $6.31 \pm 0.03$ & $5.84 \pm 0.02$ & $5.76 \pm 0.02$ \\
\hline HD 156826 & K0V & $-0.13 \pm 0.03$ & 7.17 & 6.32 & $5.02 \pm 0.02$ & $4.41 \pm 0.20$ & $4.25 \pm 0.02$ \\
\hline HD 217165 & G0 & $0.01 \pm 0.03$ & $8.25 \pm 0.01$ & $7.67 \pm 0.01$ & $6.57 \pm 0.02$ & $6.29 \pm 0.03$ & $6.19 \pm 0.02$ \\
\hline
\end{tabular}

Note. Metallicity values are derived using Spectroscopy Made Easy and taken from Valenti \& Fischer (2005). Visual $(B, V)$ magnitudes are taken from the optical photometry catalogs of Oja (1996), Jenkins et al. (2008), and Koen et al. (2010). Near-infrared $(J, H, K)$ magnitudes are from the Two Micron All Sky Survey (2MASS) catalog of point sources (Cutri et al. 2003; Skrutskie et al. 2006). The horizontal line separates systems with confirmed companions (above) versus candidate companions (below).

Observing lists were otherwise based primarily on the strength of the RV trend and distance to the star, so as to gauge whether the companion could potentially be imaged directly. The companion minimum mass $\left(M_{2}\right)$,

$$
\begin{aligned}
M_{2} & \geqslant 5.34 \times 10^{-6} M_{\odot}\left(\frac{d}{\mathrm{pc}} \frac{\rho}{\operatorname{arcsec}}\right)^{2} \\
& \times\left|\frac{\dot{v}_{r}}{\mathrm{~m} \mathrm{~s}^{-1} \mathrm{yr}^{-1}}\right| \sqrt{27} / 2,
\end{aligned}
$$

was evaluated at hypothetical angular separations, $\rho$, and for an inferred instantaneous RV acceleration, $\dot{v}_{r}$ (Liu et al. 2002). The constant, $\sqrt{27} / 2$, is the minimum value of the orbital function $F(i, e, \omega, \phi)$. The dependent values of the function are $i$ inclination, $e$ eccentricity, $\omega$ longitude of periastron, and $\phi$ orbital phase. A derivation of this function can be found in Torres (1999).

As the original goal of the program was to maximize detection efficiency by discovering as many benchmark objects as possible (for atmospheric model and evolutionary model comparison purposes), no firm cuts were made to target selection criteria to establish a uniform sample, minimize observational bias, or for other statistical reasons, such as determining the occurrence rate of planets, given a nondetection of a low-mass stellar companion or BD (Montet et al. 2014). Instead, targets were observed mainly depending on the likelihood of being detected from high-resolution imaging observations (within a threshold $\Delta \mathrm{Kmag} \geqslant 8$ and projected separation $\geqslant 10$ au relative to the primary star). Figure 1 shows the demographics of the observed companions that span this range of expected detections from high-resolution imaging. 


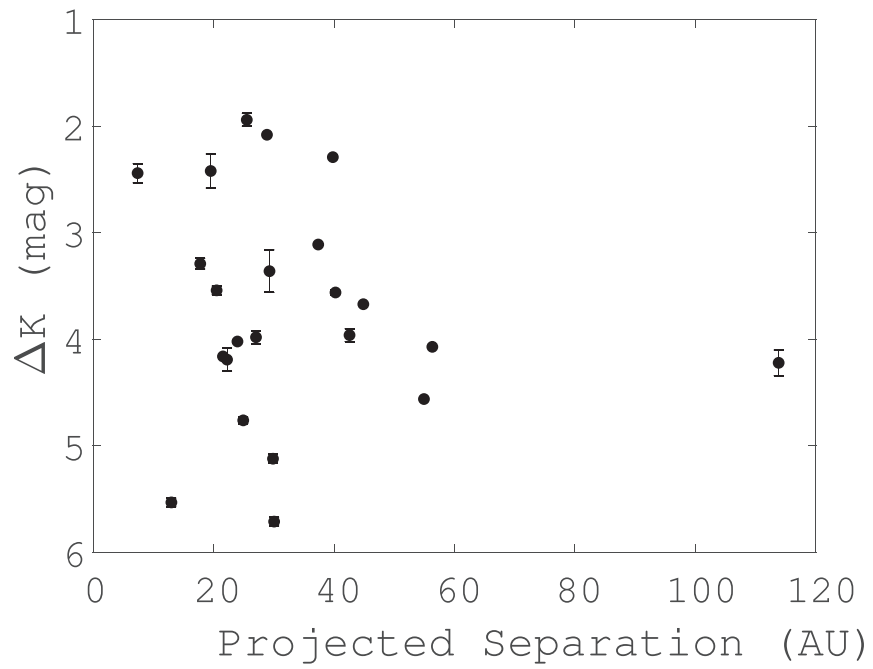

Figure 1. Demographics of compendium objects: projected physical separation of confirmed companions vs. $\Delta \mathrm{K}$ magnitude illustrating that most objects are between 20 and 100 au away from the primary star and are on long orbits.

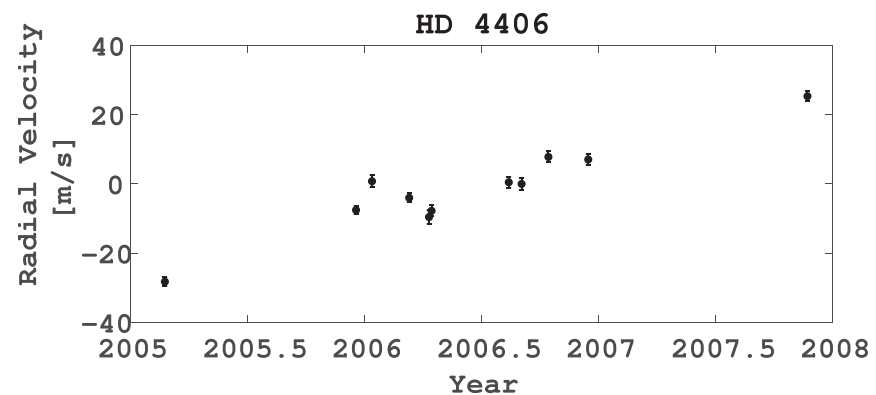

Figure 2. Radial velocity (RV) plot of HD 4406. The slope in data over time indicates the existence of a, now confirmed, companion causing the acceleration.

To further refine our sample, based on the inferred Doppler acceleration, parallax, and whether the system already showed orbital motion through the exhibition of a jerk, i.e., a change in the RV acceleration $\left(\ddot{v}_{r}=\frac{d v_{r}}{d t}\right)$, we organized follow-up imaging observations if any additional constraints on the companion angular separation and relative flux ratio could be determined a priori.

Obvious binaries, where the minimum mass from the RV time series corresponded to $M \approx 0.5 M_{\odot}$ or greater, were observed only during gaps in the $\mathrm{AO}$ schedule or poor seeing conditions. Already built into the time series velocity scatter, basic checks involving the presence of stellar noise were performed, e.g., using $R_{H K}^{\prime}$ and $v \sin i$ values as a proxy for youth, to help distinguish between accelerations caused by dynamics versus activity.

\section{Radial Velocity Observations}

Standard observing methods used by the CPS were implemented for RV observations as has been done with previous TRENDS discoveries (Howard et al. 2010). An iodine gas cell mounted in front of the spectrometer allowed for calibration of instrumental drifts and other systematics (Marcy et al. 1999). Relative RV data are visualized as plots, as exemplified in Figure 2, and are available in machine-readable form (Butler et al. 2017). Tables 3-5 are objects where the data used were not available in Butler et al. (2017). All RV plots are found in Appendix A.2. It
Table 3

Doppler RV Measurements for HD 1384

\begin{tabular}{|c|c|c|}
\hline BJD-2,440,000 & $\mathrm{RV}\left(\mathrm{m} \mathrm{s}^{-1}\right)$ & Uncertainty $\left(\mathrm{m} \mathrm{s}^{-1}\right)$ \\
\hline 14339.91 & 165.2 & 1.7 \\
\hline 14399.83 & 159.4 & 1.6 \\
\hline 14461.83 & 167.6 & 1.6 \\
\hline 14642.07 & 98.5 & 1.6 \\
\hline 14675.00 & 111.0 & 1.5 \\
\hline 14726.92 & 64.0 & 1.5 \\
\hline 14809.82 & 85.3 & 1.4 \\
\hline 14838.85 & 104.4 & 1.5 \\
\hline 14866.71 & 92.0 & 1.4 \\
\hline 14984.12 & 34.1 & 1.7 \\
\hline 15015.10 & 8.9 & 1.4 \\
\hline 15016.09 & 16.3 & 1.5 \\
\hline 15029.08 & 55.8 & 1.7 \\
\hline 15049.05 & 36.8 & 1.6 \\
\hline 15078.14 & 65.3 & 1.6 \\
\hline 15079.05 & 30.9 & 1.4 \\
\hline 15080.04 & 15.8 & 1.4 \\
\hline 15081.05 & 25.9 & 1.4 \\
\hline 15082.14 & 72.3 & 1.4 \\
\hline 15083.15 & 55.4 & 1.6 \\
\hline 15084.09 & 44.2 & 1.4 \\
\hline 15085.14 & 62.8 & 1.4 \\
\hline 15106.92 & 43.7 & 1.5 \\
\hline 15134.04 & 61.5 & 1.6 \\
\hline 15169.71 & -24.2 & 1.6 \\
\hline 15190.72 & 14.0 & 1.4 \\
\hline 15196.72 & 13.5 & 1.3 \\
\hline 15231.77 & 30.6 & 1.5 \\
\hline 15256.72 & 40.1 & 1.6 \\
\hline 15373.13 & -47.7 & 1.4 \\
\hline 15401.10 & -9.1 & 1.5 \\
\hline 15471.90 & -81.3 & 1.5 \\
\hline 15584.71 & -28.0 & 1.4 \\
\hline 16114.11 & -152.3 & 1.6 \\
\hline 16166.11 & -130.1 & 1.5 \\
\hline 16584.99 & -196.1 & 1.7 \\
\hline 16851.13 & -282.6 & 1.5 \\
\hline 16895.06 & -283.2 & 1.5 \\
\hline 18393.05 & -584.5 & 1.4 \\
\hline
\end{tabular}

Note. HD 1384 was not included in data reduction of Butler et al. (2017). The reported measurements are produced with a distinct yet similar code as described in Howard et al. (2010). This table also serves as an example of the RV data used to create the plots in Figures 7 and 8. All RV data products can be found in a machine-readable table in Butler et al. (2017).

is noted that for some of the plots, the error bars are smaller than the data points due to the high resolution of HIRES.

\section{High-contrast Imaging}

TRENDS campaign targets are generally nearby $(d \lesssim 100$ pc), bright $(V \lesssim 10)$, main-sequence stars (Tables 1 and 2 ). Reconnaissance imaging observations span several observing seasons starting as early as 2010 May. Observations were obtained in natural guide star AO mode using NIRC2 (Instrument PI: Keith Matthews) on the Keck II telescope (Wizinowich et al. 2000). Targets were first vetted for binarity using unocculted, snapshot images. ${ }^{9}$ Lower-dynamic-range

\footnotetext{
9 Additional observations for lower-priority targets were obtained using PHARO at Palomar (Hayward et al. 2001). Binaries discovered by PHARO will be reported in a separate paper as they require a different reduction pipeline and have not yet been fully processed.
} 
Table 4

Doppler RV Measurements for HD 155413

\begin{tabular}{lcc}
\hline \hline BJD-2,440,000 & RV $\left(\mathrm{m} \mathrm{s}^{-1}\right)$ & Uncertainty $\left(\mathrm{m} \mathrm{s}^{-1}\right)$ \\
\hline 13842.13 & 30.4 & 1.8 \\
13926.90 & 27.6 & 1.6 \\
13960.84 & 30.8 & 1.4 \\
13961.78 & 29.3 & 1.3 \\
13962.77 & 19.2 & 1.3 \\
13963.81 & 19.9 & 1.5 \\
13981.75 & 22.3 & 1.3 \\
13982.72 & 17.8 & 1.3 \\
13983.72 & 20.2 & 1.4 \\
15471.71 & -64.5 & 1.6 \\
16862.75 & -154.4 & 1.7 \\
\hline
\end{tabular}

Note. HD 155413 was not included in data reduction of Butler et al. (2017). The reported measurements are produced with a distinct yet similar code as described in Howard et al. (2010).

Table 5

Doppler RV Measurements for HD 4406

\begin{tabular}{lrc}
\hline \hline BJD-2,440,000 & RV $\left(\mathrm{m} \mathrm{s}^{-1}\right)$ & Uncertainty $\left(\mathrm{m} \mathrm{s}^{-1}\right)$ \\
\hline 13425.77 & -28.23 & 1.28 \\
13723.90 & -7.54 & 1.20 \\
13748.81 & 0.78 & 1.80 \\
13806.84 & -4.01 & 1.33 \\
13837.73 & -9.57 & 1.96 \\
13841.75 & -7.77 & 1.60 \\
13962.13 & 0.48 & 1.60 \\
13982.13 & 0.00 & 1.64 \\
14023.97 & 7.80 & 1.59 \\
14086.08 & 7.06 & 1.50 \\
14428.00 & 25.34 & 1.49 \\
\hline
\end{tabular}

Note. HD 4406 was not included in data reduction of Butler et al. (2017). The reported measurements are produced with a distinct yet similar code as described in Howard et al. (2010).

observations, which generally did not incorporate angular differential imaging (ADI), often used a narrow filter (e.g., $K_{\text {cont }}$ ) to prevent saturation, allowing for many targets to be observed per time such that meaningful upper limits could be placed on the mass and separation of companions prior to committing to deeper imaging observations. A three-point dither pattern was used for the quick snap-shot program to subtract the near-infrared sky background. Otherwise, the 600 mas diameter coronagraphic spot was used with ADI to search for RV trend companions that evaded detection using shallow observations. The objects reported herein do not utilize coronagraphy or ADI techniques. Such faint objects can be found in previous TRENDS publications.

As with previous TRENDS publications, the narrow mode camera setting was used $\left(9.952 \pm 0.002\right.$ mas pix $^{-1}$; Yelda et al. 2010 ) with a $1024 \times 1024$ pixel FOV. ${ }^{10}$ Standard background subtraction, flat-fielding, distortion correction, and speckle suppression methods were used to reduce science frames (Yelda et al. 2010; Crepp et al. 2012b; Service et al. 2016). Observations were generally carried out in the $K_{s}$ and $K^{\prime}$ filters.

\footnotetext{
10 The plate scale from Service et al. (2016) was used for observations following the NIRC2 hardware upgrade in 2015 April.
}

Table 6

Colors, Inferred Spectral Types, and Effective Temperatures of Companions

\begin{tabular}{|c|c|c|c|c|}
\hline (a) Name & (b) $J-K$ & (c) $H-K$ & $\begin{array}{l}\text { (d) Spec- } \\
\text { tral Type }\end{array}$ & $\begin{array}{l}\text { (e) } T_{\text {eff }} \\
(\mathrm{K})\end{array}$ \\
\hline HIP 1294 B & $0.97 \pm 0.04$ & $\ldots$ & M6.5V & 2710 \\
\hline HD $1384 \mathrm{~B}$ & $0.22 \pm 0.33$ & $0.00 \pm 0.28$ & F5V & 6510 \\
\hline HD 6512 B & $0.85 \pm 0.11$ & $\cdots$ & M4.5V & 3100 \\
\hline HD 31018 B & $\star \star$ & $0.96 \pm 0.26$ & $\ldots$ & $\cdots$ \\
\hline HD 34721 B & $1.00 \pm 0.27$ & $\cdots$ & M7V & 2650 \\
\hline HD 50639 B & $0.48 \pm 0.45$ & $\ldots$ & $\mathrm{K} 0.5 \mathrm{~V}$ & 5240 \\
\hline HD 85472 B & $0.18 \pm 0.41$ & $\ldots$ & $\mathrm{K} 3 \mathrm{~V}$ & 4830 \\
\hline HIP 55507 B & $0.93 \pm 0.13$ & $\ldots$ & M $5.5 \mathrm{~V}$ & 3000 \\
\hline \multirow{2}{*}{$110537 \mathrm{~B}^{\mathrm{a}}$} & $0.70 \pm 0.03$ & $\cdots$ & $\mathrm{K} 5 \mathrm{~V}$ & 4410 \\
\hline & $1.59 \pm 0.08$ & $\ldots$ & L3V & 1830 \\
\hline HD $111031 \mathrm{~B}$ & $0.69 \pm 0.04$ & $\ldots$ & $\mathrm{K} 5 \mathrm{~V}$ & 4410 \\
\hline HIP 63762 B & $1.02 \pm 0.09$ & $\ldots$ & M7V & 2650 \\
\hline HD 129814 B & $2.18 \pm 0.18$ & $\ldots$ & $\ldots$ & $\ldots$ \\
\hline HD 136274 B & $0.81 \pm 0.15$ & $\ldots$ & MOV & 3870 \\
\hline HD $136274 \mathrm{C}$ & $0.83 \pm 0.13$ & $\ldots$ & M4V & 3200 \\
\hline HD 139457 B & $0.68 \pm 0.19$ & $\ldots$ & $\mathrm{K} 4.5 \mathrm{~V}$ & 4540 \\
\hline HD 142229 B & $0.62 \pm 0.08$ & $\ldots$ & $\mathrm{K} 3.5 \mathrm{~V}$ & 4700 \\
\hline \multirow{3}{*}{$\begin{array}{l}\text { HD } \\
\qquad 147231 \mathrm{~B}^{\mathrm{a}}\end{array}$} & $1.55 \pm 0.04$ & $0.55 \pm 0.04$ & $\mathrm{~L} 2 \mathrm{~V}$ & 1960 \\
\hline & & & & \\
\hline & $0.72 \pm 0.04$ & $\cdots$ & $\mathrm{K} 5.5 \mathrm{~V}$ & 4330 \\
\hline HD $155413 \mathrm{~B}$ & $0.79 \pm 0.07$ & $\ldots$ & $\mathrm{K} 8 \mathrm{~V}$ & 4000 \\
\hline HD 164509 B & $0.97 \pm 0.04$ & $\ldots$ & M6.5V & 2710 \\
\hline HD $180684 \mathrm{~B}$ & $0.50 \pm 0.06$ & $\ldots$ & $\mathrm{K} 1.5 \mathrm{~V}$ & 5140 \\
\hline $\begin{array}{l}\text { HD } \\
\qquad 183473 \mathrm{~B}^{\mathrm{b}}\end{array}$ & $0.69 \pm 9.99$ & $0.03 \pm 9.99$ & M4V & 3200 \\
\hline HD $196201 \mathrm{~B}$ & $1.01 \pm 0.16$ & $0.34 \pm 0.17$ & M7V & 2650 \\
\hline HD $196201 \mathrm{C}$ & $1.41 \pm 0.13$ & $0.82 \pm 0.17$ & L2V & 1960 \\
\hline HD 201924 B & $0.80 \pm 0.04$ & $\ldots$ & $\mathrm{K} 8 \mathrm{~V}$ & 4000 \\
\hline HD 213519 B & $0.53 \pm 0.03$ & $\ldots$ & $\mathrm{K} 3.5 \mathrm{~V}$ & 4700 \\
\hline HIP 46199 B & $1.19 \pm 0.50$ & $\ldots$ & $\mathrm{M} 8.5 \mathrm{~V}$ & 2440 \\
\hline HD 131509 B & $0.86 \pm 0.06$ & $\ldots$ & M4.5V & 3100 \\
\hline HD $156826 \mathrm{~B}$ & $1.94 \pm 0.34$ & $\ldots$ & L3V & 1830 \\
\hline HD $156826 \mathrm{C}$ & $1.09 \pm 0.15$ & $\ldots$ & M7.5V & 2600 \\
\hline
\end{tabular}

Notes. $J-K$ colors are used to estimate the spectral type using the Pecaut \& Mamajek (2013) values that were most recently updated in August 2018. Colors are averaged for targets with multiple epochs or multi-filter observations unless there is large uncertainty between the two epochs ${ }^{\mathrm{a}}$. Confirmed and candidate companions are respectively listed above and below the horizontal line. Observations in filter were not obtained for this object.

${ }^{a}$ Large uncertainties in color exists between different epochs and thus we list both possible spectral types.

b The large uncertainty in HD $183473 \mathrm{~B}$ is due to the large uncertainty in $K$ magnitude of the primary star. $J-H=0.83 \pm 0.38$ for this object and is used to estimate the spectral type.

When appropriate, multi-epoch observations were recorded in complementary filters to obtain color information, assess proper motions, and begin to track orbits. Table 6 lists the dates of observations, integration times, and filters used. In some cases, small subarray camera modes were required to minimize integration time and avoid saturation of the bright onaxis star in order to calculate contrast ratios.

Figure 3 displays an example our AO images. 31 companions were found to be comoving and an additional 11 candidate companions were detected that we identify as likely to be gravitationally bound based on self-consistency arguments involving mass and projected orbital separation compared to the inferred Doppler trend (Section 6). Images of all companions can be found in Appendix A.3. Of the 31 


\section{HD 4406}

\section{$0.1 "$}

Figure 3. AO image of a confirmed binary system, HD 4406. Angular scale size legend is on the lower right hand corner.

companions proven to be associated with their parent star, six were found to be members of hierarchical triple star systems (HD 1205ABC, HD 136274ABC, and HD 196201ABC).

\section{Astrometry and Photometry}

Astrometric positioning and photometric flux values were measured relative to the primary star. For companions detected with unocculted (non-coronagraphic) AO data, standard routines were used to determine the angular separation, position angle, and relative flux value for each reduced frame (Crepp et al. 2012b). Generally, 10 to 20 images of each companion were recorded per epoch to estimate frame-to-frame statistical variance. As with previous TRENDS discoveries, statistical uncertainties and systematic effects were folded into the analysis using Monte Carlo error propagation methods (Crepp et al. 2014, 2016; Cheetham et al. 2018). For triple star systems, we used the Bayesian methods described in Bechter et al. (2014) to model the NIRC2 point-spread function, deblend sources, and determine uncertainties for each companion's astrometric position and relative flux value.

Tables in Appendix A.4 list photometric and astrometric analysis. A machine-readable table is available for the complete analysis of confirmed companions. Absolute magnitudes and projected physical separations are calculated using recent parallax measurements from Gaia (Lindegren et al. 2016; Gaia Collaboration et al. 2018). Time baselines between observations span several months to several years with an average of $2.2 \mathrm{yr}$ for confirmed companions. The high proper-motion of TRENDS targets and signal-to-noise ratio of $\mathrm{AO}$-assisted NIRC2 astrometry allows candidate companions to be evaluated for sharing common space motion with the on-axis star using only two or three epochs.

Figure 4 is an example of the space motion diagrams of each star and detected companion taking into account proper motion and parallactic motion. Each epoch of relative astrometry is plotted as a point with coordinates of DN ( $\Delta$ North) and DE ( $\Delta$ East). The track plotted in teal shows the motion of a background object, given the proper motion of the primary star. The companions data points fall off this track and thus show they are comoving with the primary star. All plots can be found in Appendix A.5.

\section{Companion Mass Estimates}

The majority of TRENDS detections are found to be bound companions (Section 5). This is due to the selection bias of

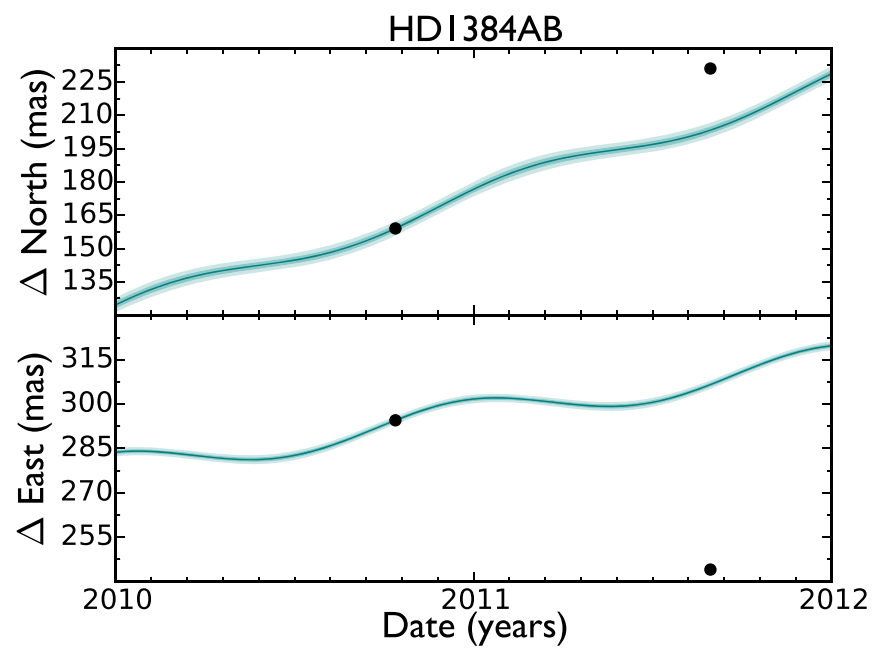

Figure 4. Example common proper motion plot showing space motion of HD $1384 \mathrm{~B}$, compared to a background object at infinity as indicated by the track plotted in teal.

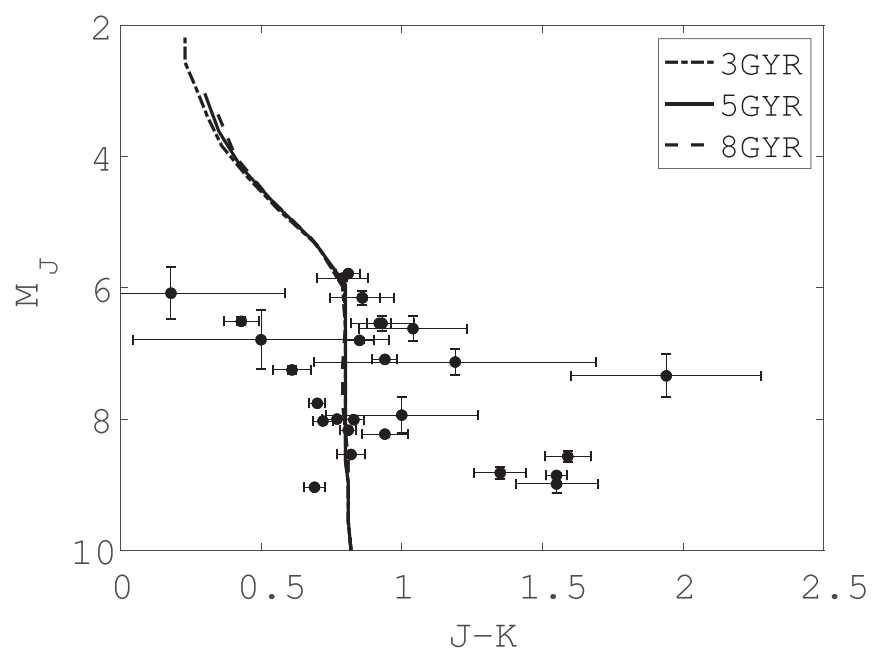

Figure 5. Color magnitude diagram: column (b) of Table 7 vs. column (d) of Tables 8 and 9. See Table 7 for inferred spectral types and effective temperatures. We have removed outliers with an error deviation greater than $3 \sigma$ of the average. We have also removed the two objects, HD 1384 and HD 129814 , in need of further spectroscopic observations and thus unreliable inferred spectral types. We overlay isochrones of 3, 5, and 8 Gyr models from Baraffe et al. (2015).

identifying stars based on the existence of RV accelerations. Of 39 stars observed to have a nearby companion, we confirm 31 companions as comoving. We further identify 11 as highly probable companions based on having only one epoch in one filter. These targets have an infrared color (Table 6, Figure 5) expected of VLM stars (Dupuy \& Liu 2012; Faherty et al. 2016).

We estimate companion masses using two methods: (i) evolutionary models that relate near-infrared absolute magnitude to mass Baraffe et al. (2015); and (ii) minimum mass limits, $M_{2}$ from Equation (1), based on the companion projected separation and inferred RV acceleration Torres (1999). A number of Doppler trends are not linear but show low levels of curvature. In either case, we evaluate the instantaneous acceleration to calculate the companion minimum mass based on Newtonian dynamics.

Using the contrast ratio between the companion and host star, we first deblend the signal(s) by correcting the apparent 


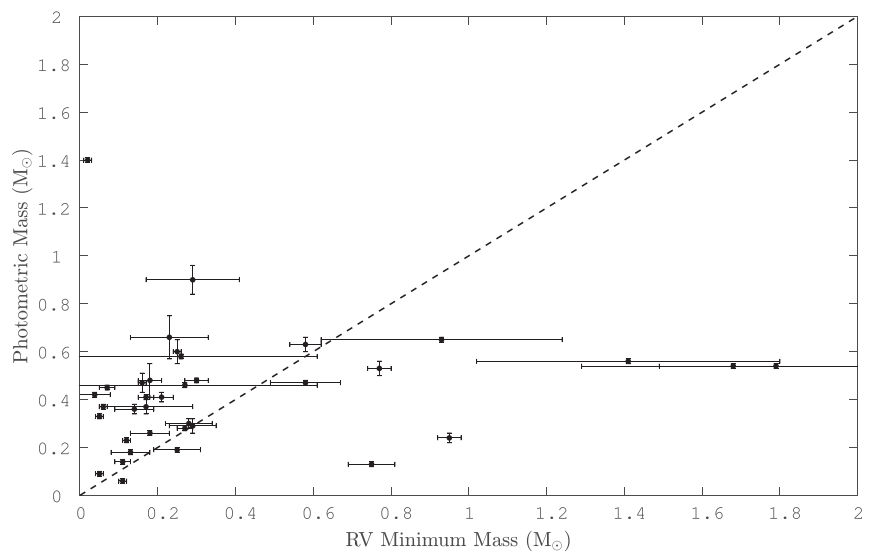

Figure 6. Comparison of inferred RV and photometric masses: columns (i) and (j) of Tables 7 and 8 are plotted to demonstrate the distribution of imaged companions in this survey. The dashed line is of the form RV Minimum Mass $=$ Photometric Mass. Objects above the dashed line represent companions responsible for the RV acceleration and expected from this survey. Objects below the dashed line represent imaged companions not responsible for the RV acceleration at the measured separation of the observed companion. See Section 7 for details on these objects.

magnitude of each source based on the system combined light. In the case of a triple star, we further deblend the apparent magnitude of the combined secondary and tertiary companions. To estimate the companion masses using evolutionary models, we interpolate model grids by Baraffe et al. (2015) across both mass and age to give a finer grid of magnitudes for each band. Using a Markov Chain Monte Carlo (MCMC) simulation, we then explore the three-dimensional (age, mass, magnitude) space including the $\mathrm{J}$-, $\mathrm{H}$-, or $\mathrm{K}$-band magnitudes and an assumed age estimate of $5 \pm 3 \mathrm{Gyr}$ as priors for each companion. We perform the MCMC simulation using emcee (Foreman-Mackey et al. 2013), a Python package which implements an affine-invariant ensemble sampler (Goodman \& Weare 2010). Table 7 lists all observational setups for TRENDS objects. Tables 8 and 9 list all astrometric and photometric measurements and the resulting inferred separations and mass measurements.

Figure 6 shows a comparison between the photometric masses based on evolutionary models and the minimum masses based on dynamics. Targets with a ratio of less than 1 are indicative of another companion that exists that is not responsible for the RV trend (see notes on specific objects in Section 7).

\section{Notes on Specific Objects}

Specific objects in this survey are of interest for future observations for the following reasons:

1. They have a confirmed companion observed that does not cause the RV acceleration as emphasized in Figure 2.

2. They have a tertiary companion previously unresolved yet a prior known binary component from previous surveys.

3 . The colors measured with this survey do not correspond to an identifiable spectral type from the extensive work done by Pecaut \& Mamajek (2013; Table 6, Figure 5) and require more detailed observations with integral field spectroscopy (IFS).

\subsection{HIP 1294}

HIP 1294 is a triple star system with an unresolved tight binary and a faint tertiary companion. We treat the tight binary as a point source and the measured separation and delta magnitude of the tertiary are with respect to the point source. At a separation of $739.69 \pm 0.92$ mas, a companion causing an $\mathrm{RV}$ acceleration of $130.22 \pm 30.14 \mathrm{~m} \mathrm{~s}^{-1} \mathrm{yr}^{-1}$ would have a minimum mass of $1.68 \pm 0.39 M_{\odot}$. The imaged tertiary located at the aforementioned separation has a photometric mass of $0.54 \pm 0.01 M_{\odot}$ and thus we asses the acceleration must be largely due to the unresolved secondary companion.

\section{2. $H D 1205$}

HD 1205 is a known binary (Høg et al. 2000). We image the known companion at $1093.2 \pm 6.29$ mas and reveal a closer companion at $216.5 \pm 4.72$ mas. The RV trend is likely due to the bright secondary that was unresolved until this survey.

\section{3. $H D 1384$}

At a separation of $335.95 \pm 4.38$ mas, a companion causing an RV acceleration of $-65.92 \pm 0.32 \mathrm{~m} \mathrm{~s}^{-1} \mathrm{yr}^{-1}$ would have a minimum mass of $2.67 \pm 0.48 M_{\odot}$. The imaged companion located at the aforementioned separation has a photometric mass of $1.03 \pm 0.07 M_{\odot}$ and thus further observations of HD 1384 are recommended. Of further interest, HD $1384 \mathrm{~B}$ has both $J-K$ and $H-K$ colors that indicate it is an F5 star with an effective temperature of $6510 \mathrm{~K}$ (Table 6, Figure 5). HD 1384 A is a G5 star (Table 2). Of the two epochs of photometric measurements, the smallest difference in magnitude between HD $1384 \mathrm{AB}$ is $\Delta K=3.32 \pm 0.06$. HD $1384 \mathrm{~B}$ is fainter and thus it is not likely to be an F5 star. Further observations with IFS are needed to determine the spectral type.

\section{4. $H D 6558$}

At a separation of $4777.50 \pm 2.93$ mas, a companion causing an RV acceleration of $-37.28 \pm 0.88 \mathrm{~m} \mathrm{~s}^{-1} \mathrm{yr}^{-1}$ would have a minimum mass of $79.38 \pm 4.92 M_{\odot}$. The imaged companion located at the aforementioned separation has a photometric mass of $0.33 \pm 0.01 M_{\odot}$. This indicates there may be a smaller, closer-in companion responsible for the trend.

\section{5. $H D 31018$}

HD $31018 \mathrm{~B}$ has an $H-K=0.96 \pm 0.26$ color. Using the Pecaut \& Mamajek (2013) table, we were unable to identify a spectral type for this object (Table 6, Figure 5). We suggest another epoch of imaging in $J, H$, and $K$ filters or follow up with IFS to determine the spectral type.

\subsection{HIP 63762}

At a separation of $1077.53 \pm 10.34$ mas, a companion causing an RV acceleration of $642.27 \pm 2.06 \mathrm{~m} \mathrm{~s}^{-1} \mathrm{yr}^{-1}$ would have a minimum mass of $6.70 \pm 0.07 M_{\odot}$. The imaged companion located at the aforementioned separation has a photometric mass of $0.28 \pm 0.01 M_{\odot}$. The RV fit is made using six data points over a $1.5 \mathrm{yr}$ baseline. Curvature is evident in these data points and may be responsible for the high estimate of the RV acceleration. Further observations of HIP 63762 are recommended.

\section{7. $H D 129814$}

HD 129814 B has a $J-K=2.18 \pm 0.18$ color. Using Pecaut $\&$ Mamajek (2013), we were unable to identify a spectral type for this object (Table 6, Figure 5). We suggest another epoch of 
imaging in $J, H$, and $K$ filters or follow up with IFS to determine the spectral type.

\section{8. $H D 136274$}

HD 136274 is a triple star system. The RV acceleration of $-31.09 \pm 0.70 \mathrm{~m} \mathrm{~s}^{-1} \mathrm{yr}^{-1}$ suggests a companion minimum mass of $0.16 \pm 0.01 M_{\odot}$ at $575.15 \pm 1.5$ mas separation. Imaging reveals a $0.47 \pm 0.04 M_{\odot}$ secondary companion at this separation. We also reveal a $0.22 \pm 0.03 M_{\odot}$ tertiary companion at $3339.14 \pm 4.03$ mas. The $\mathrm{RV}$ acceleration would require a tertiary companion of $5.58 \pm 0.26 M_{\odot}$ at the wider separation. We conclude that the RV acceleration is due to the close-in secondary and we serendipitously discover a tertiary companion.

\section{9. $H D 164509$}

Giguere et al. (2012) found a $0.48 M_{\text {Jup }}$ planet, HD $164509 \mathrm{~b}$, with an RV signature of $14.2 \pm 2.7 \mathrm{~m} \mathrm{~s}^{1}$. They allude to another companion or stellar jitter being responsible for the residual RV trend of $-5.1 \pm 0.7 \mathrm{~m} \mathrm{~s}^{-1} \mathrm{yr}^{-1}$. Ngo et al. (2017) confirm the co-movement of a VLM stellar companion and thus the binarity of the HD 164509 system. We imaged the $0.45 \pm 0.01 \odot$ companion on 2013 August 18 UT and confirmed the companion on 2016 April 21.

\subsection{0. $H D 183473$}

HD 183473 exhibits a distinct linear trend with an RV acceleration fit of $40.85 \pm 0.52 \mathrm{~m} \mathrm{~s}^{-1} \mathrm{yr}^{-1}$. At a separation of $446.52 \pm 0.76$ mas, the suggested minimum mass of a companion is $0.93 \pm 0.31 M_{\odot}$. High-resolution imaging in the $H$ and $K^{\prime}$ filters reveal a fainter companion with a suggested photometric mass of $0.65 \pm 0.01$. Due to the low-resolution magnitude of the primary star in the $K$-band, from $2 \mathrm{MASS}$, we use the $H$-band magnitude to compute a photometric mass. The imaged companion is less massive than suggested by the RV acceleration and thus further observations are suggested to determine if a fainter closer-in companion is contributing to the linear trend.

\subsection{HD 196201}

HD 196201 is a triple star system. Imaging reveals a $0.53 \pm 0.03 M_{\odot}$ secondary companion at $608.51 \pm 0.28$ mas away. We also reveal a $0.24 \pm 0.02 M_{\odot}$ tertiary companion at $657.83 \pm 0.29$ mas. We asses the RV acceleration may be due to the combined effect of the tight binary comprised of the secondary and tertiary stars.

\section{Summary and Concluding Remarks}

The TRENDS high-contrast imaging program has been active since 2010 May, and has produced several notable discoveries including two benchmark BDs and two compact objects (Crepp et al. 2012b, 2013a, 2013b, 2014, 2016, 2018). In the course of detecting and studying progressively fainter companions as AO technologies continually improve, TRENDS has uncovered several dozen stellar-mass objects in the process. This paper reports the detection of all candidate and bona-fide companions that we have imaged using Keck/ NIRC2 since the program's inception.

We find that many of the slowly varying Doppler accelerations measured as part of the Lick-Carnegie and CPS campaigns are caused by stellar companions. We have used these data sets (Figures 7 and 8) to place constraints on the companions' (Figures 9 and 10) orbits and masses from dynamics with initial comparisons to evolutionary models. Several systems have been found to be hierarchical triple stars, often with the binary components (that produce the RV acceleration) separated at the spatial resolution limit offered by Keck NGS AO.

One objective of the survey was to provide a sample of VLM stars and BD objects to follow up for future dynamical studies. Future observations obtaining astrometry of the objects reported herein, that are confirmed comoving companions to main sequence stars (Figure 11), can be used to trace the orbit of these benchmark stars and thus ascertain a dynamical mass to subsequently study the spectral energy distribution (SED) of the lower mass companions.

The next step, as shown in the mass benchmark HD 4747B (Crepp et al. 2018), IFS can be used to obtain a high-resolution SED of these VLM stars in an attempt to refine their spectral types and effective temperatures, which currently are based on color information (Figure 3). The lack of benchmark M-stars, and knowledge of M-stars and/or ability to fit their spectra, will be enhanced by mass benchmark M-stars that are companions to well-characterized $\mathrm{F}, \mathrm{G}$, and $\mathrm{K}$ stars. In addition, M-stars are the optimal targets for finding small, Earth-like planets as pursued by the current Transiting Exoplanet Survey Satellite (Ricker et al. 2015; Muirhead et al. 2018). Thus, better characterization of low-mass stars will translate to refined characterization and inferred planet parameters of planets around these small, faint host stars.

We thank Greg P. Laughlin for providing an early release of the RV data that was later produced in the manuscript by Butler et al. (2017). We thank the entire CPS team for their contribution to the legacy of RVs that inspired this work. E. J.G. thanks Ian J.M. Crossfield and Andy Skemer for their critique and support during the writing process. E.J.G. and this material is based upon work supported by the National Science Foundation Graduate Research Fellowship under grant No. 1339067. J.R.C. acknowledges support from the NASA Early Career (NNX13AB03G) and NSF CAREER (1654125) fellowship programs.

This work has made use of data from the European Space Agency (ESA) mission Gaia (https://www.cosmos.esa.int/ gaia), processed by the Gaia Data Processing and Analysis Consortium (DPAC, https://www.cosmos.esa.int/web/gaia/ dpac/consortium). Funding for the DPAC has been provided by national institutions, in particular the institutions participating in the Gaia Multilateral Agreement. We are appreciative of the vision and support of the Potenziani and Wolfe families. We are grateful to the island of Hawaii and the use of the W. M. Keck Observatory on the revered mountain of Maunakea, mahalo.

Facility: Keck:II (HIRES, NIRC2).

\section{Appendix}

\section{A.1. Table of Observations and Observational Setup}

Table 7 contains the observational dates, filter, integration time, and collecting array used with Keck/NIRC2 for each TRENDS confirmed and candidate companion. 
Table 7

Adaptive Optics Imaging Observations

\begin{tabular}{|c|c|c|c|c|c|c|c|c|c|}
\hline \multicolumn{5}{|c|}{ ve Uptics in } & \multicolumn{5}{|c|}{ (Continued) } \\
\hline Name & JD-2,440,000 & Filter & $\Delta t(\mathrm{~s})$ & $\overline{\text { Array }}$ & $\overline{\text { Name }}$ & JD-2,440,000 & Filter & $\Delta t(\mathrm{~s})$ & $\overline{\text { Array }}$ \\
\hline \multirow[t]{4}{*}{ HD $224983 \mathrm{AB}$} & 15803.8 & $K^{\prime}$ & 33.75 & 128 & \multirow{7}{*}{ HD $155413 \mathrm{AB}$} & 16054.9 & $J$ & 11.43 & 256 \\
\hline & 16522.9 & $J_{\text {cont }}$ & 126 & 1024 & & 16054.9 & $K^{\prime}$ & 11.43 & 256 \\
\hline & 16522.9 & $K_{\text {cont }}$ & 240 & 1024 & & 16076.9 & $J^{\mathrm{a}}$ & 108 & 300 \\
\hline & 17213.1 & $K_{\text {cont }}$ & 36 & 1024 & & 16076.9 & $K^{\prime}$ & 108 & 300 \\
\hline \multirow[t]{3}{*}{ HIP $1294 \mathrm{AB}$} & 15803.8 & $J$ & 37.5 & 128 & & 16522.7 & $J_{\text {cont }}$ & 180 & 1024 \\
\hline & 15803.8 & $K^{\prime}$ & 37.5 & 128 & & 16522.7 & $K_{\text {cont }}$ & 162 & 1024 \\
\hline & 16943.8 & $K_{s}$ & 45.12 & 128 & & 17171.8 & $K_{\text {cont }}$ & 27 & 1024 \\
\hline \multirow[t]{3}{*}{ HD $1205 \mathrm{ABC}$} & 16514.1 & $K_{\text {cont }}$ & 300 & 1024 & \multirow[t]{3}{*}{ HD $157338 \mathrm{AB}$} & 15803.8 & $K^{\prime}$ & 18.75 & 128 \\
\hline & 16943.9 & $K_{s}$ & 280 & 256 & & 16076.9 & $\mathrm{~J}^{\mathrm{a}}$ & 25 & 300 \\
\hline & 17213.1 & $K_{\text {cont }}$ & 198 & 1024 & & 16076.9 & $K^{\prime}$ & 25 & 300 \\
\hline \multirow[t]{4}{*}{ HD $1384 \mathrm{AB}$} & 15482.8 & $J$ & 1.2 & 128 & \multirow[t]{3}{*}{ HD $164509 \mathrm{AB}$} & 16522.8 & $K_{\text {cont }}$ & 180 & 1024 \\
\hline & 15482.8 & $H$ & 1.2 & 128 & & 17500.0 & $J_{\text {cont }}$ & 100 & 1024 \\
\hline & 15482.8 & $K^{\prime}$ & 1.2 & 128 & & 17500.0 & $K_{\text {cont }}$ & 100 & 1024 \\
\hline & 15803.8 & $K^{\prime}$ & 101.25 & 1024 & HD $180684 \mathrm{AB}$ & 16055.1 & $J$ & 13 & 140 \\
\hline $\mathrm{HD} 6512 \mathrm{AB}$ & 16523.1 & $J_{\text {cont }}$ & 180 & 1024 & & 16055.1 & $K^{\prime}$ & 13 & 140 \\
\hline & 16523.1 & $K_{\text {cont }}$ & 150 & 1024 & & 17500.5 & $J_{\text {cont }}$ & 54 & 1024 \\
\hline & 16943.8 & $K_{\text {cont }}$ & 54.3 & 1024 & & 17500.5 & $K_{\text {cont }}$ & 54 & 1024 \\
\hline HD $31018 \mathrm{AB}$ & 15483.5 & $J^{\mathrm{a}}$ & 2.25 & 128 & HD $183473 \mathrm{AB}$ & 15342.1 & $\mathrm{H}$ & .5 & 512 \\
\hline & 15483.5 & $K^{\prime}$ & 1.125 & 128 & & 16102.9 & $J$ & 22.58 & 128 \\
\hline & 15483.5 & $L^{\prime \mathrm{a}}$ & 1.125 & 128 & & 16102.9 & $\mathrm{H}$ & 22.58 & 128 \\
\hline & 15933.9 & $H$ & 20.25 & 128 & & 16102.9 & $K^{\prime}$ & 35.25 & 256 \\
\hline & 15933.9 & $K^{\prime \mathrm{a}}$ & 15 & 1024 & HD $196201 \mathrm{ABC}$ & 15803.8 & $J$ & 37.5 & 128 \\
\hline & 15933.9 & $K^{\prime}$ & 20.25 & 128 & & 15803.8 & $H$ & 37.5 & 128 \\
\hline HD $34721 \mathrm{AB}$ & 16943.9 & $J_{\text {cont }}{ }^{\mathrm{a}}$ & 150 & 1024 & & 15803.8 & $K^{\prime}$ & 37.5 & 128 \\
\hline & 16943.9 & $K_{\text {cont }}$ & 150 & 1024 & & 16055.1 & $J$ & 45 & 256 \\
\hline & 17290.1 & $J_{\text {cont }}$ & 106 & 512 & & 16055.1 & $K^{\prime}$ & 45 & 256 \\
\hline & 17290.1 & $K_{\text {cont }}$ & 106 & 512 & & 16942.8 & $K_{\text {cont }}$ & 200 & 1024 \\
\hline $\mathrm{HD} 40647 \mathrm{AB}$ & 15482.1 & $K^{\prime}$ & 2.25 & 1024 & HD $201924 \mathrm{AB}$ & 16113.0 & $J$ & 38.125 & 192 \\
\hline & 15933.9 & $K^{\prime}$ & 18 & 256 & & 16113.0 & $K^{\prime}$ & 38.125 & 256 \\
\hline HD $50639 \mathrm{AB}$ & 16222.1 & $J$ & 34.3 & 256 & & 16165.9 & $\mathrm{~J}$ & 14.5 & 256 \\
\hline & 16222.1 & $K^{\prime}$ & 34.3 & 256 & & 16165.9 & $K^{\prime}$ & 14.5 & 256 \\
\hline & 17291.2 & $J^{\mathrm{a}}$ & 200 & 256 & HD $213519 \mathrm{AB}$ & 15803.9 & $\mathrm{~J}^{\mathrm{a}}$ & 10 & 1024 \\
\hline & 17291.2 & $K_{s}$ & 200 & 256 & & 15803.9 & $K^{\prime}$ & 67.5 & 128 \\
\hline HD $85472 \mathrm{AB}$ & 16054.8 & $J$ & 18.75 & 128 & & 15803.9 & $K^{\prime}$ & 10 & 1024 \\
\hline & 16054.8 & $K_{s}$ & 18.75 & 128 & & 16103.0 & $J$ & 18 & 256 \\
\hline & 16403.8 & $J^{\mathrm{a}}$ & 22.5 & 128 & & 16103.0 & $K^{\prime}$ & 18 & 256 \\
\hline & $\begin{array}{l}16403.8 \\
159341\end{array}$ & $\begin{array}{l}K_{s} \\
K^{\prime}\end{array}$ & $\begin{array}{c}22.5 \\
7.5\end{array}$ & $\begin{array}{l}128 \\
256\end{array}$ & HD $1293 \mathrm{AB}$ & 15483.8 & $K^{\prime \mathrm{a}}$ & 1.125 & 128 \\
\hline HIP 55507 AB & $\begin{array}{l}15934.1 \\
17171.7\end{array}$ & $J_{\text {cont }}^{\Lambda}$ & 150 & 1024 & & 15483.8 & $\mathrm{H}$ & 1.125 & 128 \\
\hline & 17171.7 & $K_{\text {cont }}$ & 100 & 1024 & & 15483.8 & $J^{\mathrm{a}}$ & 1.125 & 128 \\
\hline $\mathrm{HD} 110537 \mathrm{AB}$ & 15960.1 & $J$ & 50 & 256 & HD $1388 \mathrm{AB}$ & 15803.9 & $H^{\mathrm{a}}$ & 90 & 512 \\
\hline & 15960.1 & $K^{\prime}$ & 50 & 256 & & 15803.9 & $K^{\prime \mathrm{a}}$ & 100 & 512 \\
\hline & 17499.9 & $J_{\text {cont }}$ & 54 & 1024 & & 16514.1 & $J_{\text {cont }}{ }^{\mathrm{a}}$ & 100 & 1024 \\
\hline & 17499.9 & $K_{\text {cont }}$ & 54 & 1024 & & 16514.1 & $K_{\text {cont }}$ & 300 & 1024 \\
\hline $\mathrm{HD} 111031 \mathrm{AB}$ & 15934.1 & $K^{\prime}$ & 7.5 & 128 & HD $4406 \mathrm{ABC}$ & 15481.9 & $H$ & 1.2 & 128 \\
\hline & 17499.9 & $J_{\text {cont }}$ & 54 & 1024 & & 15481.9 & $H^{\mathrm{a}}$ & 18.1 & 1024 \\
\hline & 17499.9 & $K_{\text {cont }}$ & 54 & 1024 & & 15803.9 & $J^{\mathrm{a}}$ & 56.25 & 128 \\
\hline HIP $63762 \mathrm{AB}$ & 16076.8 & $J$ & 5.3 & 512 & & 15803.9 & $K^{\prime}$ & 56.25 & 128 \\
\hline & 16076.8 & $K^{\prime}$ & 5.3 & 512 & HD $6558 \mathrm{AB}$ & 15788.9 & $K^{\prime \mathrm{a}}$ & 33.75 & 128 \\
\hline & 17171.8 & $K_{\text {cont }}$ & 60 & 512 & & 15788.9 & $K^{\prime}$ & 45 & 512 \\
\hline HD $129191 \mathrm{AB}$ & 16054.9 & $J^{\mathrm{a}}$ & 17.6 & 256 & & 15788.9 & $K^{\prime \mathrm{a}}$ & 2490 & 1024 \\
\hline & 16054.9 & $K^{\prime}$ & 17.6 & 256 & & 15933.7 & $K^{\prime \mathrm{a}}$ & 12 & 256 \\
\hline & 16493.8 & $K_{\text {cont }}$ & 270 & 1024 & & 15933.7 & $K^{\prime \mathrm{a}}$ & 1470 & 1024 \\
\hline HD $129814 \mathrm{AB}$ & 15615.9 & $K^{\prime}$ & 175 & 512 & HD 88986 AB & 15934.1 & $K^{\prime}$ & 13 & 156 \\
\hline & 16054.9 & $J$ & 18 & 256 & & 16403.8 & $\mathrm{~K}_{s}^{\mathrm{a}}$ & 11.25 & 1024 \\
\hline & 16054.9 & $K^{\prime}$ & 18 & 256 & HIP 46199 AB & 16403.8 & $J$ & 15 & 128 \\
\hline & 17171.8 & $K_{\text {cont }}$ & 180 & 1024 & & 16403.8 & $K^{\prime}$ & 15 & 128 \\
\hline HD $136274 \mathrm{ABC}$ & 16503.8 & $J_{\text {cont }}$ & 180 & 1024 & HD 103829 AB & 17171.8 & $K_{\text {cont }}$ & 270 & 1024 \\
\hline & 16503.8 & $K_{\text {cont }}$ & 135 & 1024 & HD 105618 AB & 17171.9 & $K_{\text {cont }}$ & 300 & 1024 \\
\hline & 17171.8 & $K_{\text {cont }}$ & 45 & 1024 & HD $131509 \mathrm{AB}$ & 17171.8 & $J_{\text {cont }}$ & 187.5 & 1024 \\
\hline HD $139457 \mathrm{AB}$ & 15615.1 & $K^{\prime}$ & 5 & 512 & & 17171.8 & $K_{\text {cont }}$ & 150 & 1024 \\
\hline & 16054.9 & $J$ & 15 & 224 & HD 156826 ABC & 17171.9 & $K_{\text {cont }}$ & 75 & 512 \\
\hline & 16054.9 & $K^{\prime}$ & 15 & 224 & & 17171.9 & $J_{\text {cont }}$ & 75 & 512 \\
\hline HD $142229 \mathrm{AB}$ & 15615.1 & $K^{\prime}$ & 10 & 512 & HD $217165 \mathrm{AB}$ & 17213.1 & $K_{\text {cont }}$ & 150 & 1024 \\
\hline & 16076.8 & $J$ & 52.7 & 512 & & & & & \\
\hline & 16076.8 & $K^{\prime}$ & 52.7 & 512 & Note. & & & & \\
\hline & 17171.8 & $K_{\text {cont }}$ & 45 & 1024 & ${ }^{\mathrm{a}}$ Companion not $\mathrm{r}$ & filter, bad data, & all of a & ng area, & lagraph \\
\hline HD $147231 \mathrm{AB}$ & 15803.8 & $H$ & 37.5 & 128 & used and occulted & n. Confirmed an & date cor & n observa & listed \\
\hline & 15803.8 & $J$ & 37.5 & 128 & respectively above & w the horizonta & & & \\
\hline & 15803.8 & $K^{\prime}$ & 33.75 & 128 & 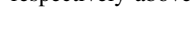 & & & & \\
\hline
\end{tabular}

Table 7

(Continued)

used and occulted companion. Confirmed and candidate companion observations are listed 


\section{A.2. Astrometry, Photometry, and Inferred Physical Measurements}

Table 8 contains astrometric measurements and the inferred resulting spatial measurements, photometric measurements, the inferred companion photometry and photometric mass measurements, and the RV acceleration and inferred RV minimum mass measurements. Table 9 contains the aforementioned measurements for candidate companions (those with one epoch of observations). Figure 6 depicts the comparison of the mass measurements and inferences made.

Table 8

Astrometry and Photometry: Confirmed Companions

\begin{tabular}{|c|c|c|c|c|c|c|c|c|c|}
\hline (a) Name & $\begin{array}{l}\text { (b) } \\
\text { Filter }\end{array}$ & (c) $\Delta \mathrm{mag}$ & (d) $M_{\text {comp }}$ & (e) $\rho$ (mas) & $(\mathrm{f})^{\mathrm{a}}$ P.A. $\left({ }^{\circ}\right)$ & $\begin{array}{l}\text { (g) Proj. } \\
\text { Sep. (au) }\end{array}$ & (h) $\dot{v}\left(\mathrm{~ms}^{-1} \mathrm{yr}^{-1}\right)$ & $(\mathrm{i})^{\mathrm{b}} M_{\min }\left(M_{\odot}\right)$ & (j) $M_{\text {phot }}\left(M_{\odot}\right)$ \\
\hline \multirow{4}{*}{ HD $224983 \mathrm{AB}$} & $K^{\prime}$ & $\mathrm{c}$ & $\mathrm{c}$ & $4640.90 \pm 6.91$ & $346.30 \pm 0.09$ & $157.19 \pm 0.40$ & $-1.75 \pm 0.35$ & $0.56 \pm 0.34$ & $\mathrm{c}$ \\
\hline & $J_{\text {cont }}$ & $3.20 \pm 0.50$ & $\mathrm{c}$ & $4660.99 \pm 1.27$ & $346.18 \pm 0.02$ & $157.87 \pm 0.33$ & $\ldots$ & $\ldots$ & $\mathrm{c}$ \\
\hline & $K_{\text {cont }}$ & $2.64 \pm 0.19$ & $\mathrm{c}$ & $4658.88 \pm 1.08$ & $346.23 \pm 0.02$ & $157.80 \pm 0.33$ & $\ldots$ & $\ldots$ & c \\
\hline & $K_{\text {cont }}$ & $2.78 \pm 0.08$ & $\mathrm{c}$ & $4603.12 \pm 2.33$ & $350.38 \pm 0.10$ & $155.91 \pm 0.33$ & $\ldots$ & $\ldots$ & c \\
\hline \multirow[t]{3}{*}{ HIP 1294 AB } & $J$ & $2.48 \pm 0.02$ & $6.54 \pm 0.03$ & $750.73 \pm 0.86$ & $98.18 \pm 0.28$ & $29.37 \pm 0.62$ & $141.32 \pm 16.08$ & $1.68 \pm 0.39$ & $0.51 \pm 0.01$ \\
\hline & $K^{\prime}$ & $2.14 \pm 0.02$ & $5.62 \pm 0.03$ & $747.34 \pm 0.21$ & $98.14 \pm 0.01$ & $29.36 \pm 0.14$ & $\ldots$ & $\ldots$ & $0.53 \pm 0.01$ \\
\hline & $K_{s}$ & $2.02 \pm 0.03$ & $5.52 \pm 0.03$ & $720.97 \pm 0.25$ & $104.16 \pm 0.01$ & $28.33 \pm 0.14$ & $\cdots$ & $\cdots$ & $0.54 \pm 0.01$ \\
\hline \multirow{3}{*}{$\mathrm{HD} 1205 \mathrm{AB}$} & $K_{\text {cont }}$ & $1.38 \pm 0.01$ & $3.97 \pm 0.11$ & $215.7 \pm 1.2$ & $213.15 \pm 0.68$ & $16.52 \pm 0.09$ & $108.65 \pm 0.82$ & $0.29 \pm 0.12$ & $0.85 \pm 0.03$ \\
\hline & $K_{s}$ & $0.96 \pm 0.03$ & $3.65 \pm 0.11$ & $219.4 \pm 4.2$ & $242.22 \pm 0.50$ & $16.78 \pm 0.10$ & $\cdots$ & $\ldots$ & $0.92 \pm 0.04$ \\
\hline & $K_{\text {cont }}$ & $0.94 \pm 0.03$ & $3.64 \pm 0.11$ & $214.4 \pm 1.8$ & $240.19 \pm 0.50$ & $16.42 \pm 0.09$ & $\cdots$ & $\ldots$ & $0.92 \pm 0.04$ \\
\hline \multirow[t]{3}{*}{ HD 1205 AC } & $K_{\text {cont }}$ & $4.01 \pm 0.02$ & $6.35 \pm 0.11$ & $1100.8 \pm 2.2$ & $165.53 \pm 0.68$ & $84.29 \pm 0.09$ & $\cdots$ & $7.74 \pm 3.17$ & $0.41 \pm 0.02$ \\
\hline & $K_{s}$ & $3.47 \pm 0.03$ & $5.83 \pm 0.12$ & $1091.7 \pm 5.3$ & $197.09 \pm 0.50$ & $83.59 \pm 0.09$ & $\cdots$ & $\ldots$ & $0.49 \pm 0.02$ \\
\hline & $K_{\text {cont }}$ & $3.39 \pm 0.02$ & $5.75 \pm 0.11$ & $1087.1 \pm 2.5$ & $195.17 \pm 0.50$ & $83.24 \pm 0.09$ & $\cdots$ & $\ldots$ & $0.51 \pm 0.02$ \\
\hline \multirow[t]{4}{*}{ HD $1384 \mathrm{AB}$} & $J$ & $2.99 \pm 0.34$ & $3.41 \pm 0.32$ & $336.1 \pm 0.7$ & $134.87 \pm 0.68$ & $60.01 \pm 0.05$ & $-65.92 \pm 0.32$ & $2.67 \pm 0.48$ & $1.05 \pm 0.08$ \\
\hline & $H$ & $3.26 \pm 0.28$ & $3.19 \pm 0.27$ & $336.9 \pm 1.1$ & $133.93 \pm 0.68$ & $59.89 \pm 0.06$ & $\ldots$ & $\cdots$ & $1.03 \pm 0.08$ \\
\hline & $K^{\prime}$ & $3.32 \pm 0.06$ & $3.13 \pm 0.08$ & $334.7 \pm 4.0$ & $134.63 \pm 0.68$ & $60.09 \pm 0.07$ & $\cdots$ & -- & $1.04 \pm 0.05$ \\
\hline & $K^{\prime}$ & $3.45 \pm 0.01$ & $3.25 \pm 0.05$ & $336.1 \pm 1.4$ & $133.44 \pm 0.09$ & $60.34 \pm 0.07$ & $\cdots$ & $\cdots$ & $1.01 \pm 0.05$ \\
\hline \multirow[t]{3}{*}{ HD $6512 \mathrm{AB}$} & $J_{\text {cont }}$ & $2.86 \pm 0.12$ & $6.15 \pm 0.11$ & $738.34 \pm 3.12$ & $86.37 \pm 0.18$ & $39.18 \pm 0.55$ & $-10.41 \pm 0.41$ & $0.21 \pm 0.03$ & $0.56 \pm 0.02$ \\
\hline & $K_{\text {cont }}$ & $2.27 \pm 0.02$ & $5.29 \pm 0.03$ & $739.11 \pm 1.48$ & $86.97 \pm 0.12$ & $40.26 \pm 0.16$ & $\ldots$ & $\ldots$ & $0.58 \pm 0.01$ \\
\hline & $K_{\text {cont }}$ & $2.30 \pm 0.01$ & $5.31 \pm 0.02$ & $721.91 \pm 0.29$ & $88.04 \pm 0.04$ & $39.32 \pm 0.13$ & $\cdots$ & $\cdots$ & $0.58 \pm 0.01$ \\
\hline \multirow[t]{3}{*}{ HD $31018 \mathrm{AB}$} & $K^{\prime}$ & $3.36 \pm 0.35$ & $4.47 \pm 0.34$ & $287.32 \pm 1.33$ & $65.03 \pm 0.27$ & $28.94 \pm 0.22$ & $18.58 \pm 1.39$ & $0.23 \pm 0.10$ & $0.74 \pm 0.08$ \\
\hline & $H$ & $4.26 \pm 0.18$ & $5.43 \pm 0.18$ & $293.11 \pm 1.59$ & $77.61 \pm 0.08$ & $29.76 \pm 0.27$ & $\ldots$ & $\ldots$ & $0.58 \pm 0.03$ \\
\hline & $K^{\prime}$ & $3.35 \pm 0.04$ & $4.46 \pm 0.04$ & $293.87 \pm 1.27$ & $77.48 \pm 0.06$ & $29.60 \pm 0.22$ & $\ldots$ & $\cdots$ & $0.73 \pm 0.01$ \\
\hline \multirow[t]{3}{*}{ HD $34721 \mathrm{AB}$} & $K_{\text {cont }}$ & $4.33 \pm 0.01$ & $6.94 \pm 0.02$ & $2176.0 \pm 5.7$ & $132.26 \pm 0.05$ & $54.45 \pm 0.01$ & $1.26 \pm 0.10$ & $0.05 \pm 0.01$ & $0.31 \pm 0.01$ \\
\hline & $J_{\text {cont }}$ & $4.71 \pm 0.02$ & $7.94 \pm 0.27$ & $2163.6 \pm 4.3$ & $131.60 \pm 0.68$ & $46.65 \pm 0.33$ & $\ldots$ & $\ldots$ & $0.29 \pm 0.04$ \\
\hline & $K_{\text {cont }}$ & $4.10 \pm 0.02$ & $6.71 \pm 0.03$ & $2159.4 \pm 4.1$ & $131.57 \pm 0.68$ & $46.56 \pm 0.33$ & $\cdots$ & $\cdots$ & $0.35 \pm 0.01$ \\
\hline \multirow[t]{2}{*}{$\mathrm{HD} 40647 \mathrm{AB}$} & $K^{\prime}$ & $2.40 \pm 0.09$ & $6.36 \pm 0.08$ & $254.86 \pm 1.76$ & $223.45 \pm 0.38$ & $8.00 \pm 0.07$ & $169.90 \pm 0.90$ & $0.17 \pm 0.02$ & $0.41 \pm 0.01$ \\
\hline & $K^{\prime}$ & $2.48 \pm 0.09$ & $6.43 \pm 0.08$ & $214.80 \pm 0.54$ & $270.11 \pm 0.24$ & $6.74 \pm 0.04$ & $\ldots$ & $\ldots$ & $0.40 \pm 0.01$ \\
\hline \multirow[t]{3}{*}{ HD 50639 AB } & $J$ & $3.66 \pm 0.47$ & $6.79 \pm 0.45$ & $489.79 \pm 1.19$ & $10.61 \pm 0.68$ & $18.65 \pm 0.17$ & $-86.98 \pm 0.77$ & $0.38 \pm 0.04$ & $0.47 \pm 0.07$ \\
\hline & $K^{\prime}$ & $3.51 \pm 0.06$ & $6.29 \pm 0.06$ & $490.99 \pm 1.06$ & $9.56 \pm 0.55$ & $19.12 \pm 0.06$ & $\cdots$ & $\cdots$ & $0.42 \pm 0.01$ \\
\hline & $K_{s}$ & $3.56 \pm 0.01$ & $6.34 \pm 0.02$ & $560.9 \pm 4.2$ & $21.96 \pm 0.45$ & $20.29 \pm 0.14$ & $\ldots$ & $\cdots$ & $0.41 \pm 0.01$ \\
\hline \multirow[t]{3}{*}{ HD $85472 \mathrm{AB}$} & $J$ & $4.48 \pm 0.41$ & $6.08 \pm 0.40$ & $323.83 \pm 0.43$ & $101.18 \pm 0.68$ & $21.45 \pm 0.07$ & $-37.17 \pm 0.30$ & $0.30 \pm 0.03$ & $0.58 \pm 0.06$ \\
\hline & $K_{s}$ & $4.74 \pm 0.03$ & $5.90 \pm 0.07$ & $325.44 \pm 0.35$ & $100.93 \pm 0.08$ & $24.73 \pm 0.06$ & $\ldots$ & $\cdots$ & $0.48 \pm 0.01$ \\
\hline & $K^{\prime}$ & $4.78 \pm 0.02$ & $5.94 \pm 0.06$ & $329.99 \pm 0.29$ & $109.88 \pm 0.04$ & $25.08 \pm 0.06$ & $\cdots$ & $\cdots$ & $0.48 \pm 0.01$ \\
\hline \multirow[t]{3}{*}{ HIP 55507 AB } & $K^{\prime}$ & $5.78 \pm 0.05$ & $10.36 \pm 0.05$ & $473.04 \pm 1.44$ & $178.48 \pm 0.07$ & $12.07 \pm 0.04$ & $24.14 \pm 0.74$ & $0.05 \pm 0.01$ & $0.08 \pm 0.01$ \\
\hline & $J_{\text {cont }}$ & $5.45 \pm 0.12$ & $10.78 \pm 0.12$ & $547.62 \pm 2.38$ & $175.31 \pm 0.31$ & $12.26 \pm 0.06$ & $\cdots$ & $\cdots$ & $0.09 \pm 0.01$ \\
\hline & $K_{\text {cont }}$ & $5.27 \pm 0.02$ & $9.85 \pm 0.03$ & $544.19 \pm 2.24$ & $174.90 \pm 0.31$ & $13.88 \pm 0.06$ & $\cdots$ & $\cdots$ & $0.09 \pm 0.01$ \\
\hline \multirow[t]{4}{*}{ HD $110537 \mathrm{AB}$} & $J$ & $4.44 \pm 0.01$ & $7.76 \pm 0.02$ & $1246.54 \pm 1.43$ & $69.76 \pm 0.15$ & $56.28 \pm 0.14$ & $7.15 \pm 0.12$ & $0.28 \pm 0.06$ & $0.31 \pm 0.01$ \\
\hline & $K^{\prime}$ & $4.11 \pm 0.01$ & $7.06 \pm 0.02$ & $1247.80 \pm 1.22$ & $67.75 \pm 0.11$ & $56.34 \pm 0.14$ & $\cdots$ & $\cdots$ & $0.29 \pm 0.02$ \\
\hline & $J_{\text {cont }}$ & $5.25 \pm 0.08$ & $8.57 \pm 0.08$ & $1283.8 \pm 3.5$ & $87.60 \pm 0.68$ & $50.57 \pm 0.53$ & $\ldots$ & $\cdots$ & $0.21 \pm 0.01$ \\
\hline & $K_{\text {cont }}$ & $4.03 \pm 0.01$ & $6.98 \pm 0.02$ & $1288.6 \pm 7.1$ & $87.72 \pm 0.68$ & $50.76 \pm 0.72$ & $\ldots$ & $\ldots$ & $0.30 \pm 0.01$ \\
\hline \multirow[t]{3}{*}{ HD $111031 \mathrm{AB}$} & $K^{\prime}$ & $5.91 \pm 0.06$ & $8.76 \pm 0.07$ & $961.77 \pm 2.11$ & $282.84 \pm 0.05$ & $30.04 \pm 0.08$ & $7.8 \pm 0.48$ & $0.11 \pm 0.02$ & $0.13 \pm 0.01$ \\
\hline & $J_{\text {cont }}$ & $5.77 \pm 0.01$ & $9.04 \pm 0.02$ & $1036.0 \pm 4.5$ & $303.99 \pm 0.68$ & $28.23 \pm 0.30$ & $\cdots$ & $\cdots$ & $0.16 \pm 0.01$ \\
\hline & $K_{\text {cont }}$ & $5.50 \pm 0.01$ & $8.35 \pm 0.03$ & $1040.9 \pm 2.8$ & $304.42 \pm 0.68$ & $28.37 \pm 0.23$ & $\cdots$ & $\cdots$ & $0.15 \pm 0.01$ \\
\hline
\end{tabular}

Notes.

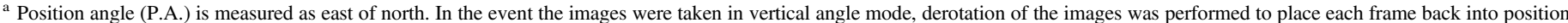
angle mode.

b SPOCS catalog used to obtain stellar mass measurement of the primary star and infer the dynamical minimum mass of the companion.

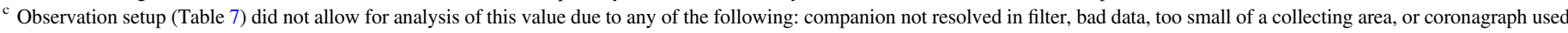
and occulted companion.

(This table is available in its entirety in machine-readable form.) 
Table 9

Astrometry and Photometry: Candidate Companions

\begin{tabular}{|c|c|c|c|c|c|c|c|c|c|}
\hline (a) Name & (b) Filter & (c) $\Delta \mathrm{mag}$ & (d) $M_{\text {comp }}$ & (e) $\rho$ (mas) & $\begin{array}{l}(\mathrm{f})^{\mathrm{a}} \text { P.A. }\left({ }^{\circ}\right) \\
\end{array}$ & ("g) Proj. Sep. (au) & (h) $\dot{v}\left(\mathrm{~m} \mathrm{~s}^{-1} \mathrm{yr}^{-1}\right)$ & $\left({ }^{(\mathrm{i})^{\mathrm{b}}} M_{\min }\left(M_{\odot}\right)\right.$ & (j) $M_{\text {phot }}\left(M_{\odot}\right)$ \\
\hline HD $1293 \mathrm{AB}$ & $H$ & $3.05 \pm 0.12$ & $3.08 \pm 0.14$ & $126.77 \pm 1.84$ & $185.20 \pm 0.61$ & $24.74 \pm 0.99$ & $68.56 \pm 1.13$ & $0.57 \pm 0.43$ & $1.05 \pm 0.06$ \\
\hline HD 1388 AB & $K_{\text {cont }}$ & $5.87 \pm 0.22$ & $8.71 \pm 0.22$ & $1677.18 \pm 2.40$ & $319.70 \pm 0.09$ & $45.20 \pm 0.09$ & $26.10 \pm 0.25$ & $0.75 \pm 0.06$ & $0.13 \pm 0.01$ \\
\hline $\mathrm{HD} 4406 \mathrm{AB}$ & $K^{\prime}$ & $0.43 \pm 0.01$ & $1.33 \pm 0.03$ & $75.96 \pm 4.98$ & $246.79 \pm 3.77$ & $9.46 \pm 0.63$ & $18.20 \pm 1.68$ & $0.02 \pm 0.01$ & $1.40 \pm 0.01$ \\
\hline HD 6558 AB & $K^{\prime}$ & $4.54 \pm 0.01$ & $6.84 \pm 0.02$ & $4777.50 \pm 2.93$ & $286.20 \pm 0.06$ & $392.23 \pm 1.93$ & $-37.28 \pm 0.88$ & $79.38 \pm 4.92$ & $0.33 \pm 0.01$ \\
\hline \multirow[t]{2}{*}{ HIP 46199 АВ } & $J$ & $1.79 \pm 0.23$ & $7.13 \pm 0.20$ & $411.4 \pm 1.2$ & $288.69 \pm 0.68$ & $8.08 \pm 0.60$ & $-152.10 \pm 2.21$ & $0.18 \pm 0.03$ & $0.41 \pm 0.03$ \\
\hline & $K^{\prime}$ & $1.16 \pm 0.62$ & $5.94 \pm 0.46$ & $412.3 \pm 1.0$ & $288.56 \pm 0.68$ & $8.10 \pm 0.60$ & $\ldots$ & $\ldots$ & $0.48 \pm 0.07$ \\
\hline HD 88986 AB & $K^{\prime}$ & $4.28 \pm 0.01$ & $6.57 \pm 0.02$ & $1521.11 \pm 1.66$ & $59.74 \pm 0.09$ & $50.65 \pm 0.08$ & $-1.65 \pm 0.07$ & $0.06 \pm 0.01$ & $0.37 \pm 0.01$ \\
\hline HD $105618 \mathrm{AB}$ & $K_{\text {cont }}$ & $5.20 \pm 0.10$ & $7.99 \pm 0.10$ & $1040.08 \pm 3.99$ & $316.75 \pm 0.22$ & $73.05 \pm 0.38$ & $3.36 \pm 0.40$ & $0.25 \pm 0.06$ & $0.19 \pm 0.01$ \\
\hline \multirow[t]{2}{*}{ HD $131509 \mathrm{AB}$} & $J_{\text {cont }}$ & $4.26 \pm 0.03$ & $6.15 \pm 0.04$ & $589.07 \pm 1.08$ & $35.78 \pm 0.14$ & $45.63 \pm 0.20$ & $-7.73 \pm 0.10$ & $0.26 \pm 0.35$ & $0.57 \pm 0.01$ \\
\hline & $K_{\text {cont }}$ & $3.95 \pm 0.05$ & $5.29 \pm 0.05$ & $598.75 \pm 0.46$ & $35.99 \pm 0.06$ & $46.38 \pm 0.18$ & $\cdots$ & $\cdots$ & $0.58 \pm 0.01$ \\
\hline \multirow[t]{2}{*}{ HD $156826 \mathrm{AB}$} & $J_{\text {cont }}$ & $5.71 \pm 0.33$ & $7.34 \pm 0.33$ & $1720.28 \pm 2.92$ & $338.78 \pm 0.04$ & $82.16 \pm 0.21$ & $-9.80 \pm 0.16$ & $1.41 \pm 0.39$ & $0.38 \pm 0.05$ \\
\hline & $K_{\text {cont }}$ & $4.53 \pm 0.07$ & $5.40 \pm 0.07$ & $1721.39 \pm 0.47$ & $338.77 \pm 0.01$ & $82.21 \pm 0.16$ & $\cdots$ & $\ldots$ & $0.56 \pm 0.01$ \\
\hline \multirow[t]{2}{*}{ HD 156828 AC } & $J_{\text {cont }}$ & $4.97 \pm 0.13$ & $6.61 \pm 0.13$ & $1939.54 \pm 2.10$ & $341.91 \pm 0.04$ & $92.63 \pm 0.20$ & $\cdots$ & $1.79 \pm 0.40$ & $0.50 \pm 0.02$ \\
\hline & $K_{\text {cont }}$ & $4.65 \pm 0.08$ & $5.52 \pm 0.08$ & $1940.01 \pm 0.66$ & $341.88 \pm 0.01$ & $92.66 \pm 0.18$ & $\ldots$ & $\cdots$ & $0.54 \pm 0.01$ \\
\hline HD $217165 \mathrm{AB}$ & $K_{\text {cont }}$ & $2.98 \pm 0.01$ & $6.02 \pm 0.02$ & $318.76 \pm 0.37$ & $73.88 \pm 0.17$ & $14.02 \pm 0.04$ & $-365.60 \pm 0.54$ & $0.27 \pm 0.34$ & $0.46 \pm 0.01$ \\
\hline
\end{tabular}

Notes. Astrometry and photometry for all candidate companions.

${ }^{\text {a }}$ Position angle (P.A) is measured as east of north. In the event the images were taken in vertical angle mode, derotation of the images was performed to place each frame back into position angle mode.

${ }^{\mathrm{b}}$ SPOCS catalog used to obtain stellar mass measurement of the primary star and infer the dynamical minimum mass of the companion.

(This table is available in its entirety in machine-readable form.) 


\section{A.3. Relative Radial Velocity Plots of Compendium Objects}

Figure 7 displays the relative RV data as plots of RV measurements over time. The slope in data over time indicates the existence of a confirmed companion causing the acceleration. See notes on special objects found in Section 7. Figure $8(a)$ is a continuation of Figure 7. Figure 8(b) displays RV data as plots for candidate companions.
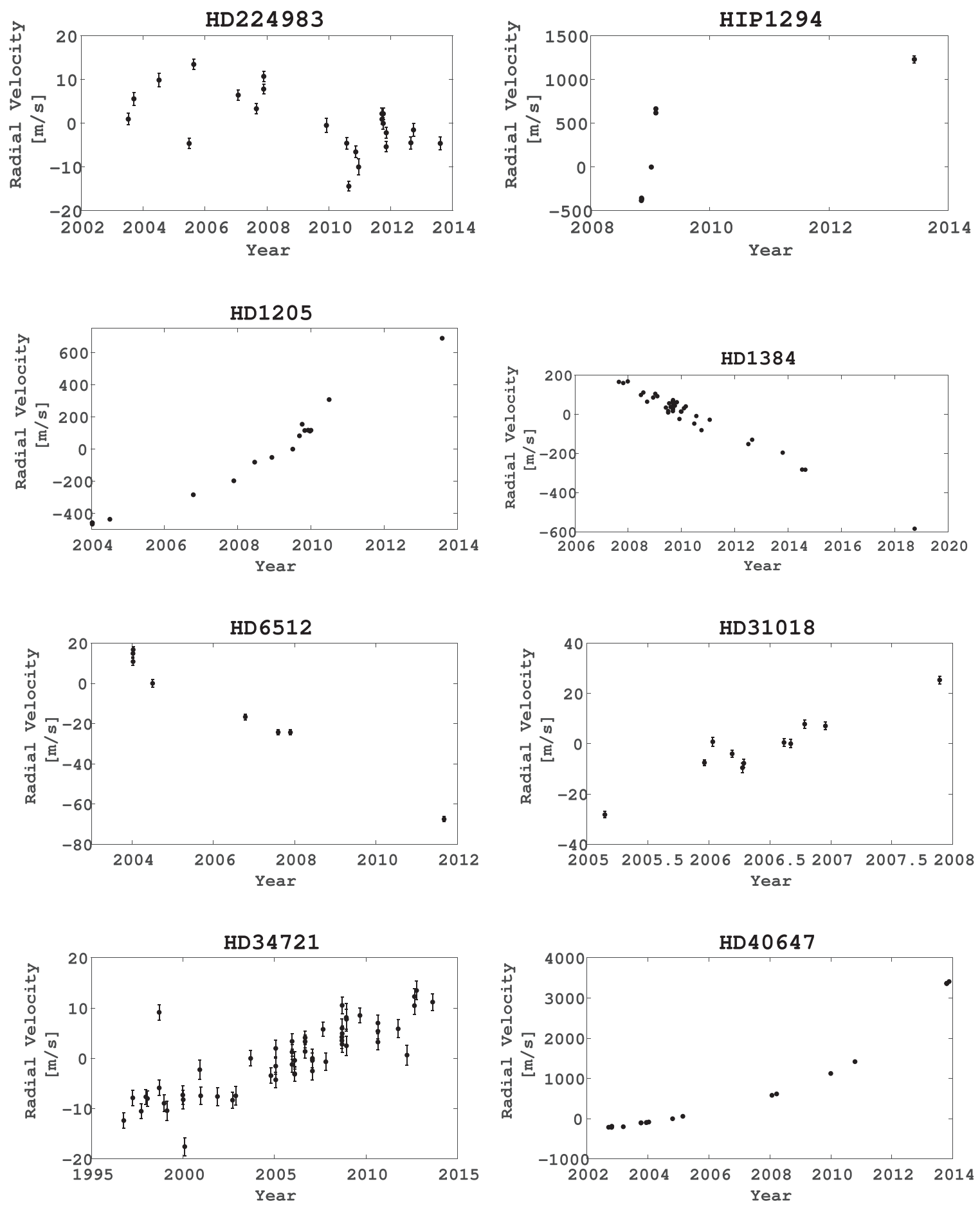

Figure 7. Relative RV plots for stars with confirmed companions. 

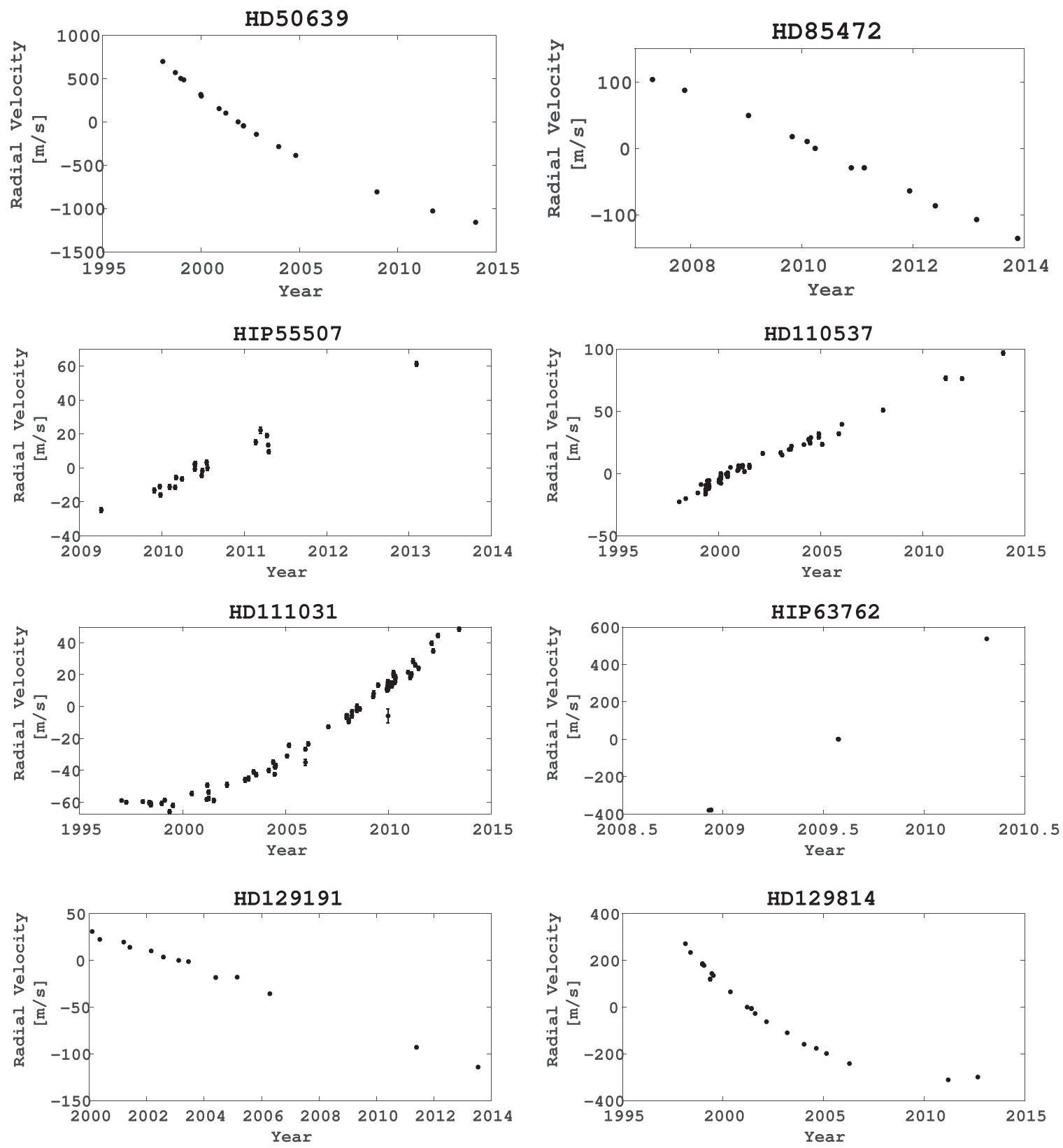

Figure 7. (Continued.) 

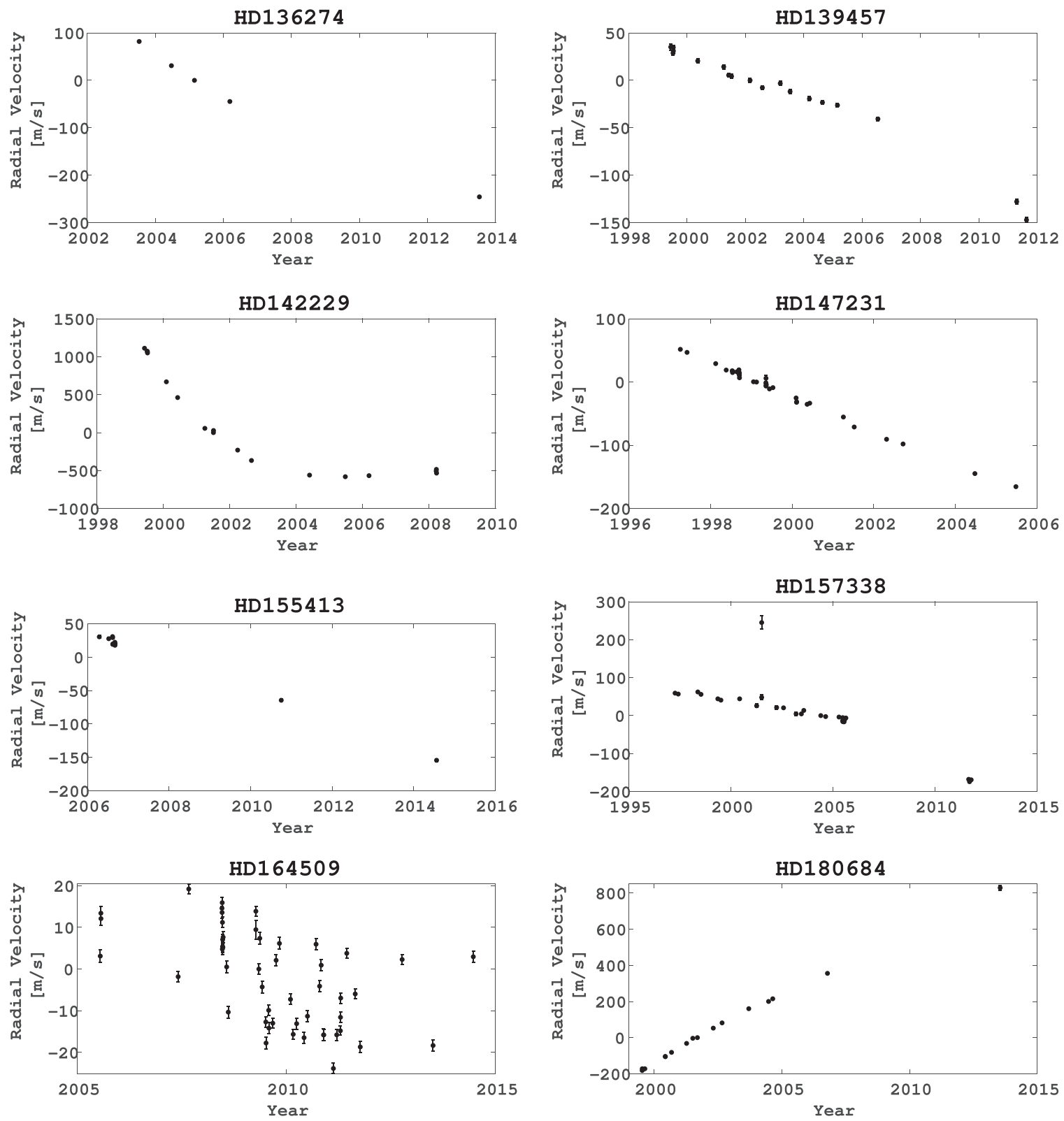

Figure 7. (Continued.) 

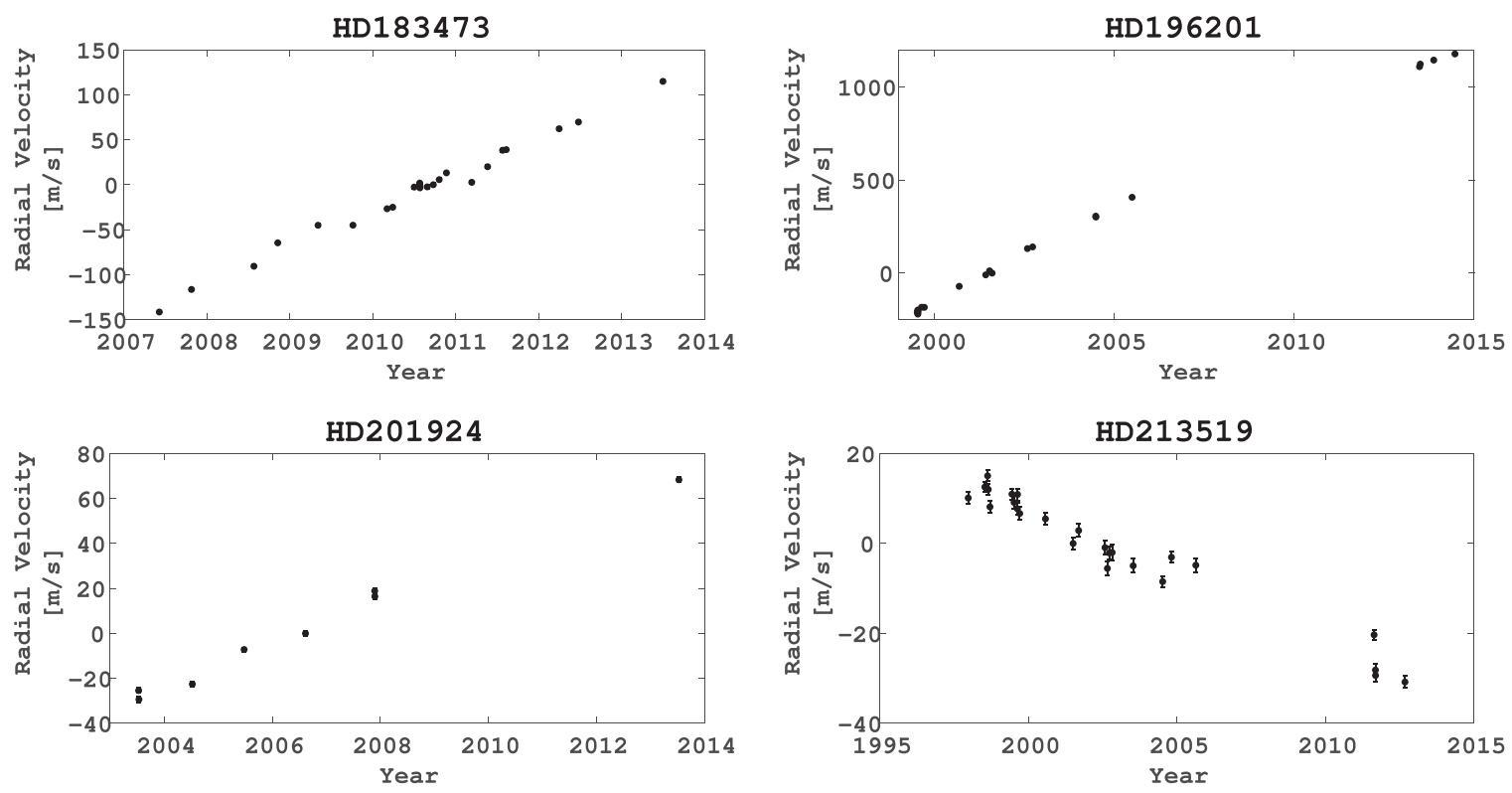

Figure 7. (Continued.) 
(a)
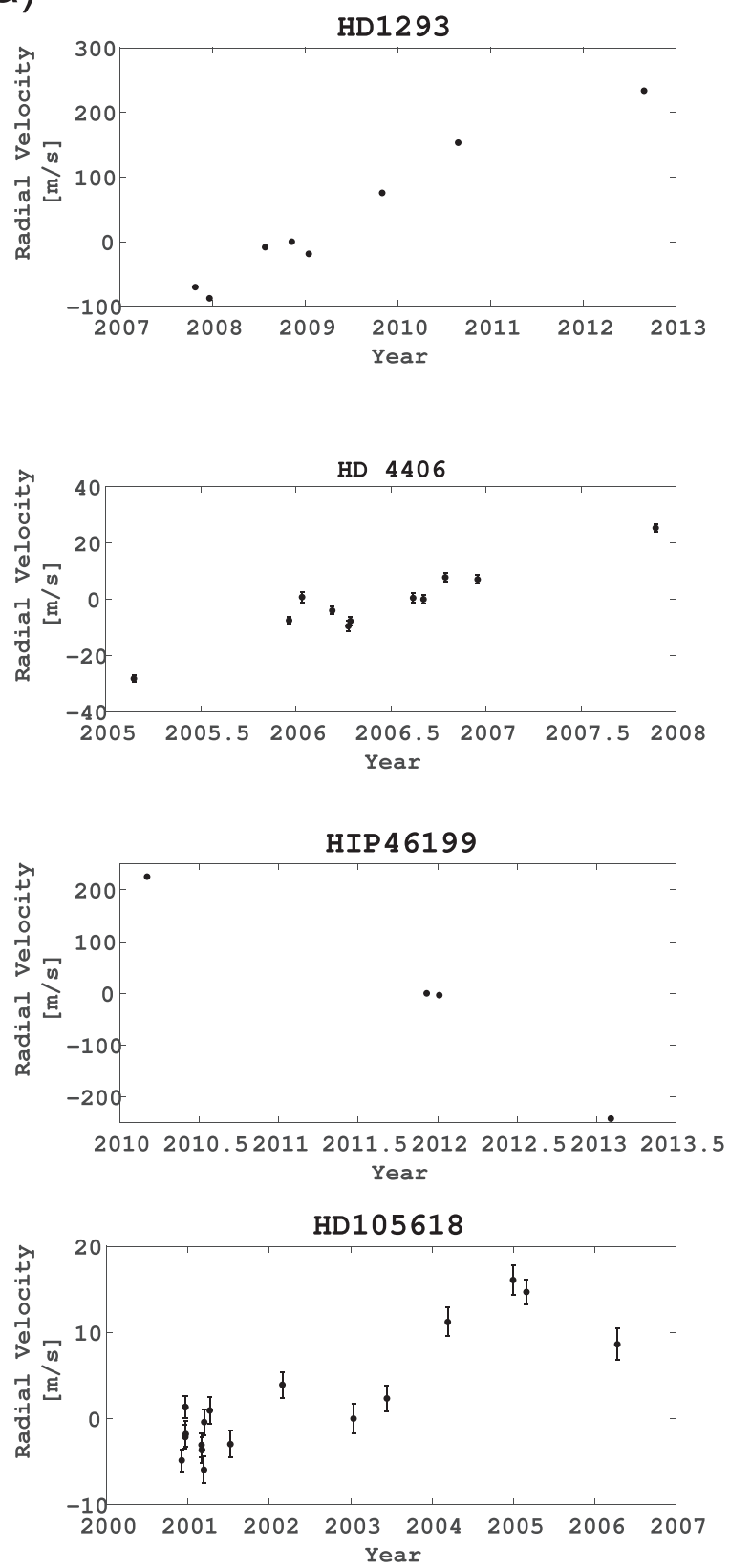

(b)

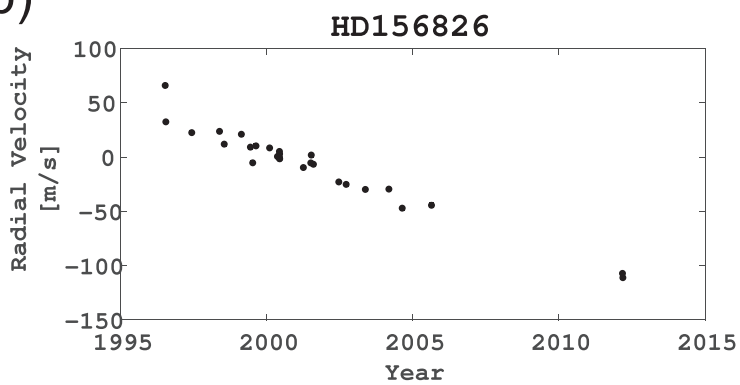

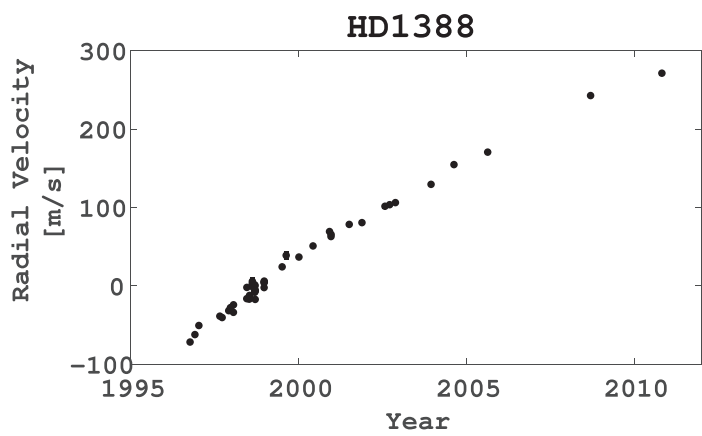
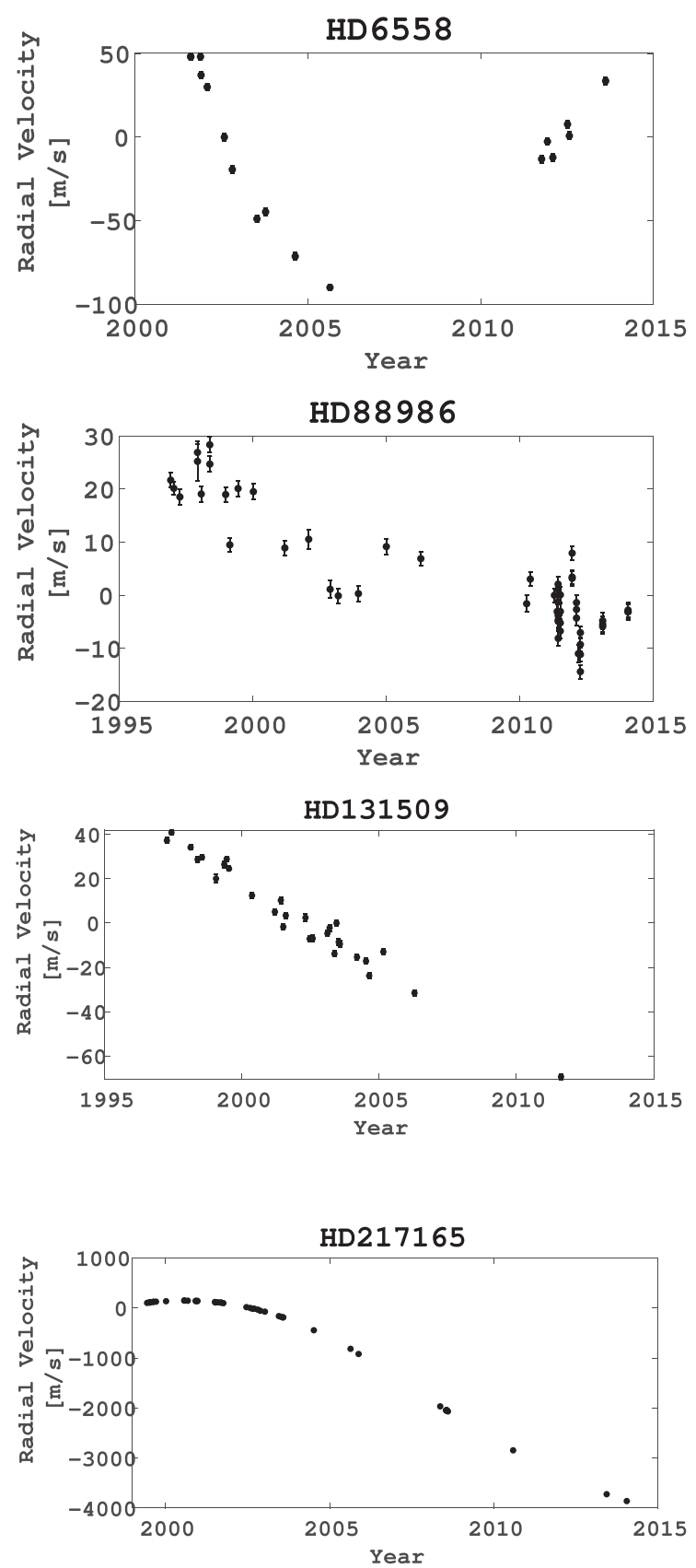

Figure 8. (a) Relative RV plots for stars with candidate companions. (b) Relative RV plots for stars with confirmed companions. 


\section{A.4. High-contrast Images of Compendium Objects}

Figure 9 contains images of confirmed companions from this survey. Figure 10 contains candidate companions from this survey.
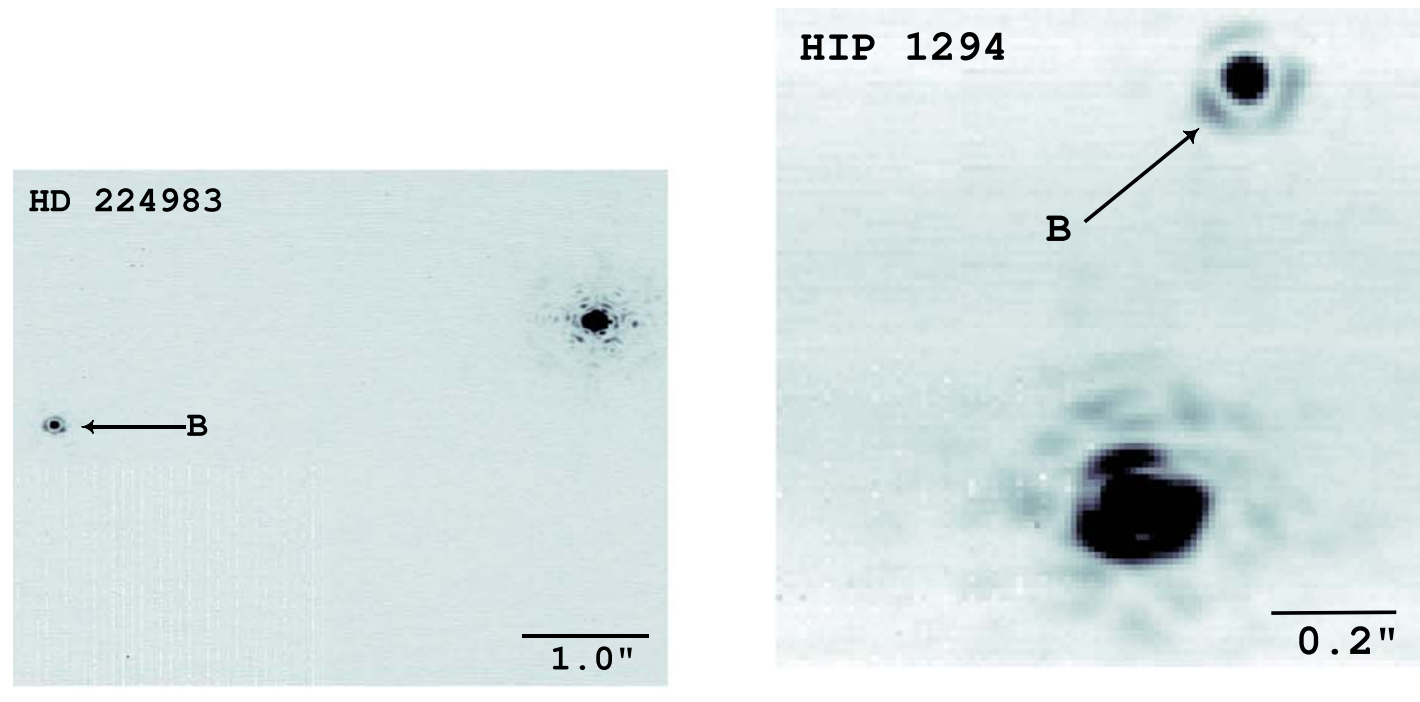

HD 1205

HD 1384
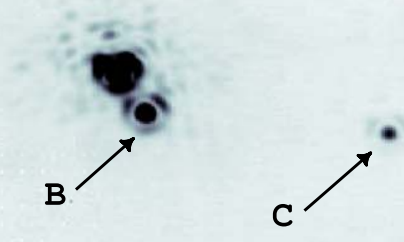

$0.5 "$

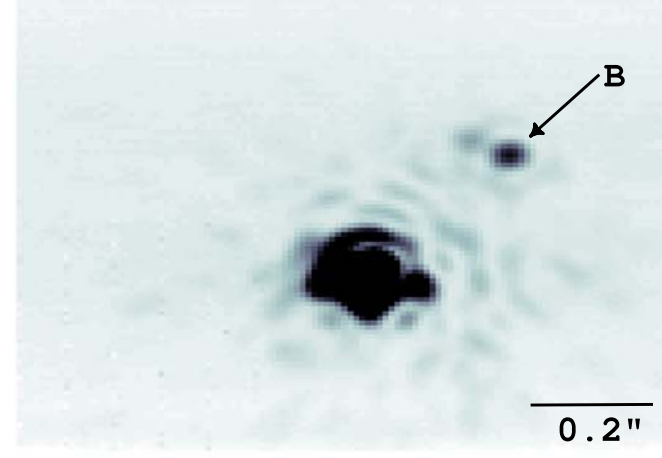

HD 6512

HD 31018
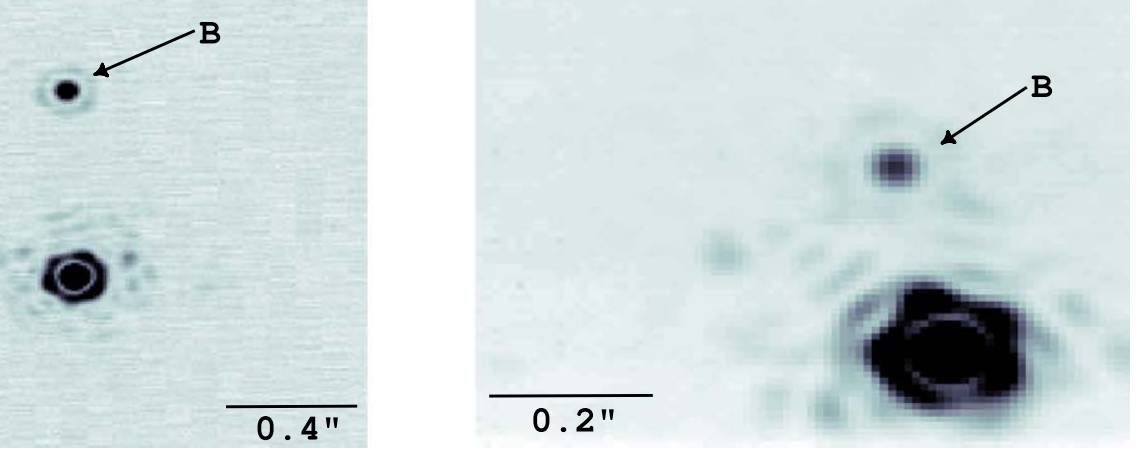

Figure 9. High-resolution adaptive optics (AO) images of confirmed companions. 

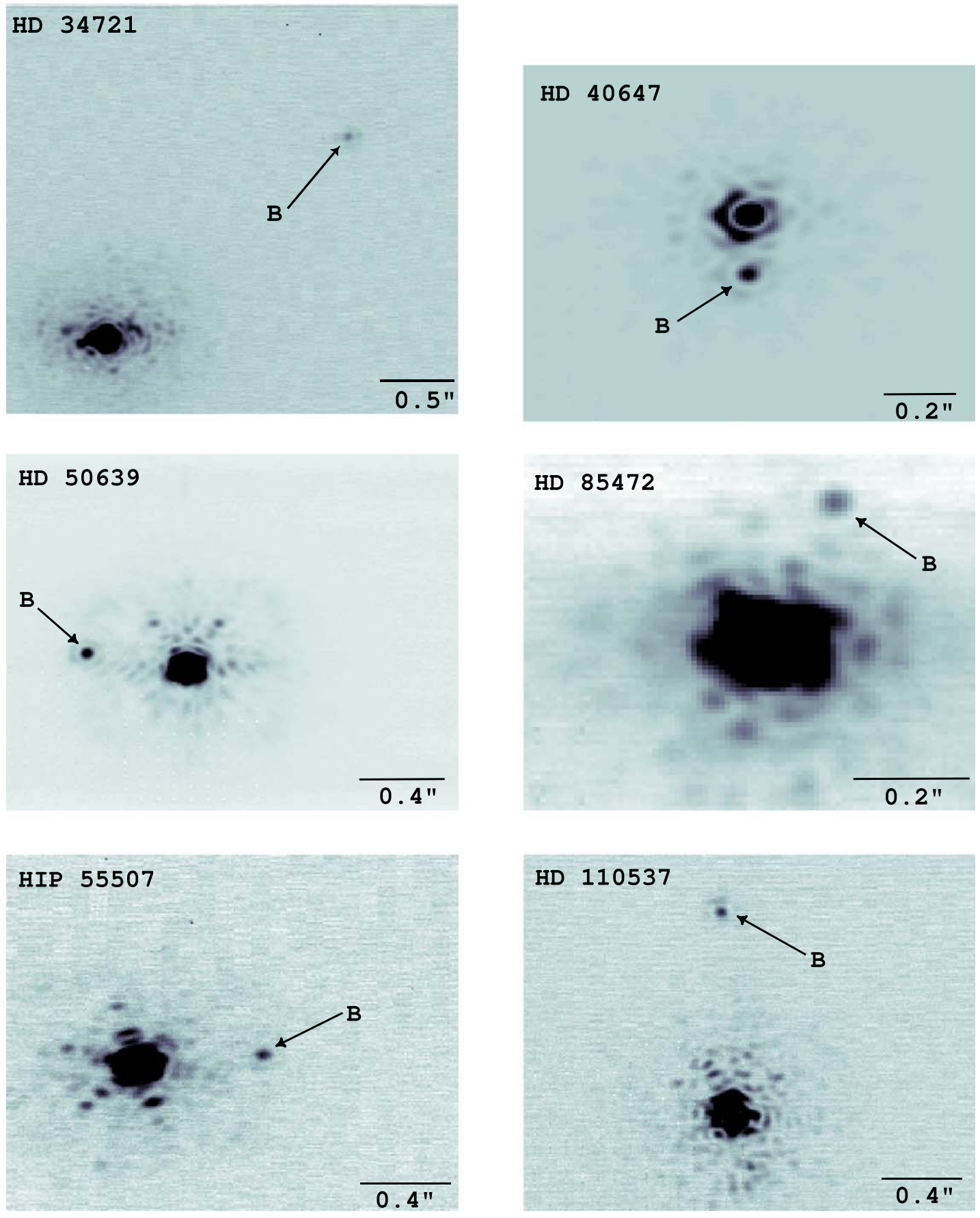

Figure 9. (Continued.) 

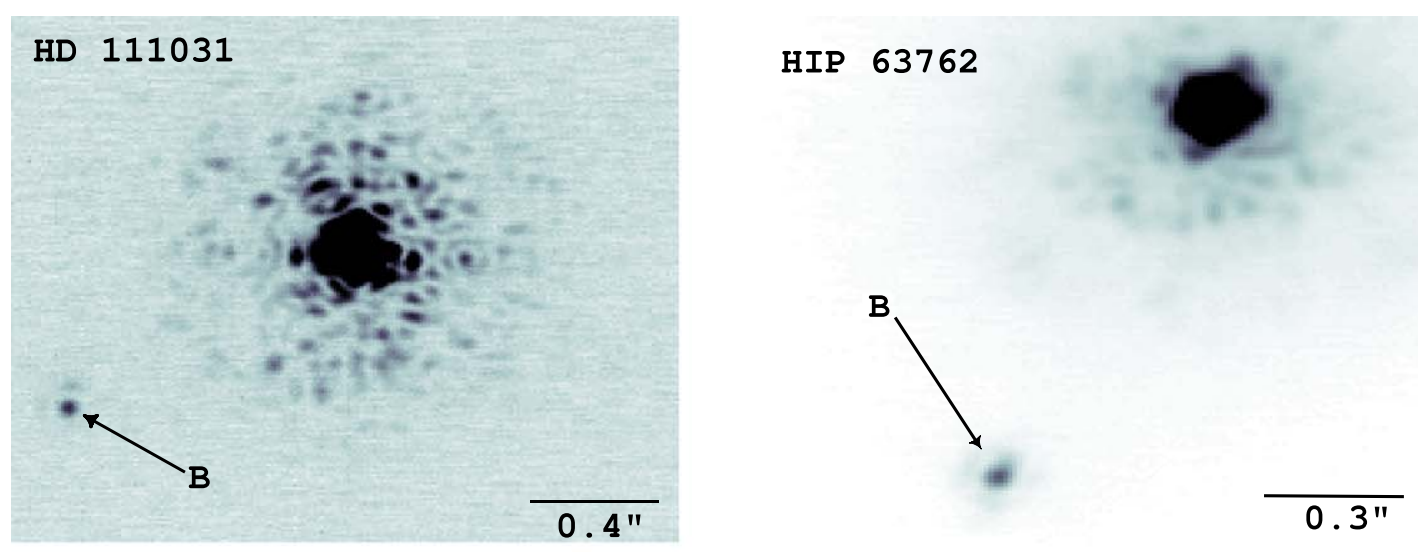

HD 129191

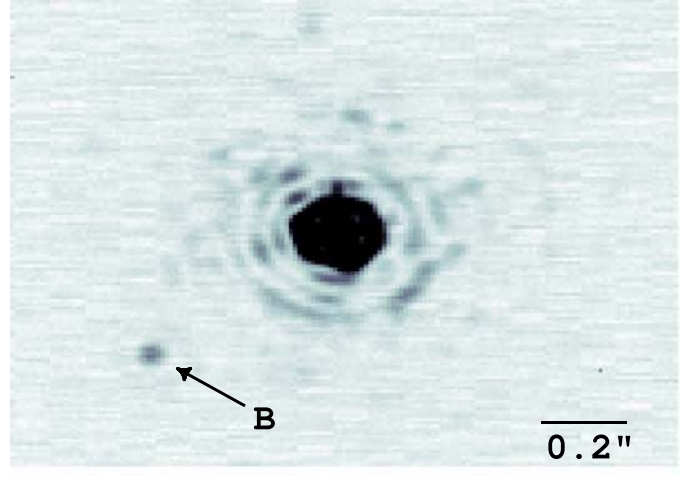

HD 136274

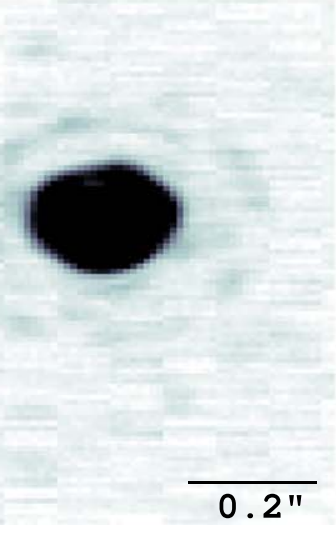

HD 129814

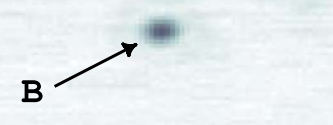

$\overline{0.2 "}$

HD 139457

B

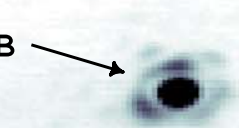

Figure 9. (Continued.) 
HD 142229

$\overline{0.2 "}$

HD 155413

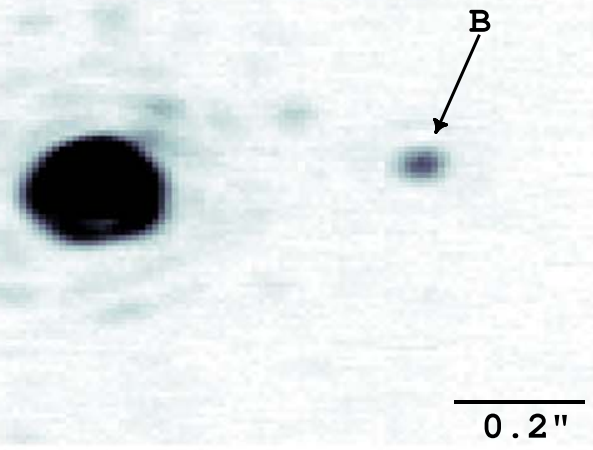

HD 164509

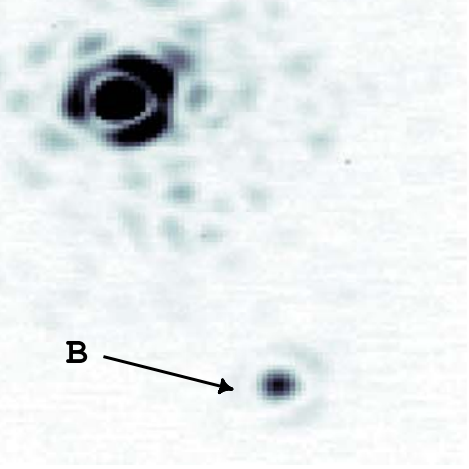

HD 147231
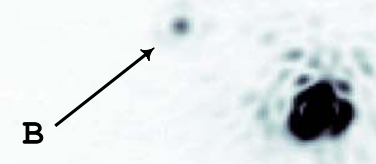

HD 157338

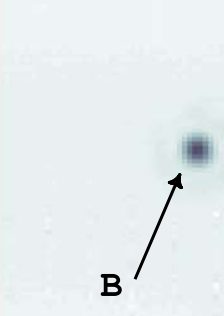

$0.4 "$

HD 180684

$\overline{0.3 "}$

Figure 9. (Continued.) 


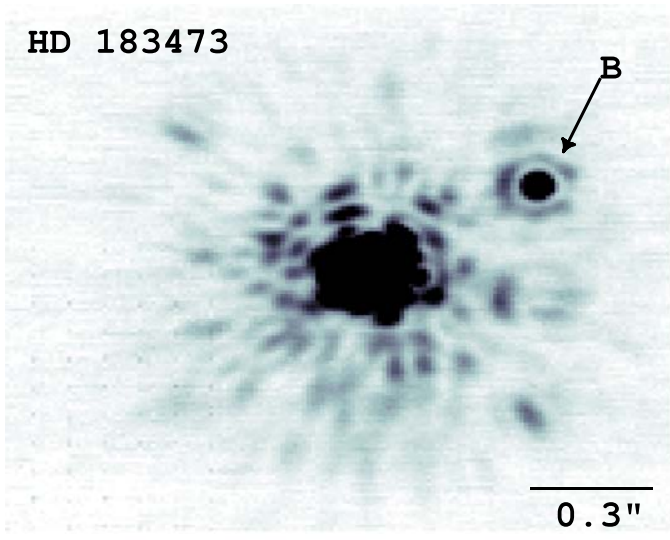

HD 196201
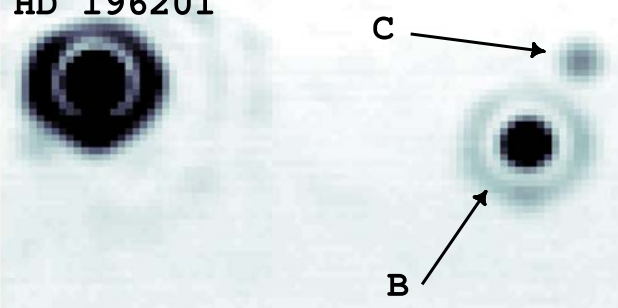

HD 201924

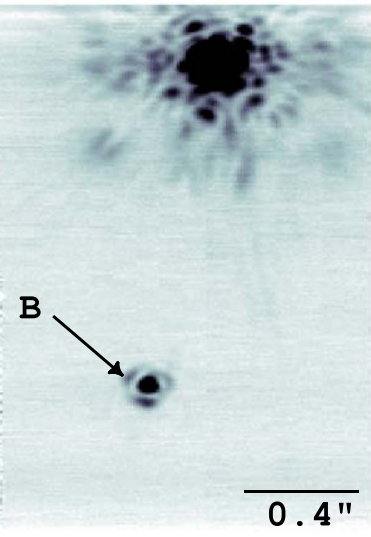

HD 213519

$0.3^{\prime \prime}$

Figure 9. (Continued.) 


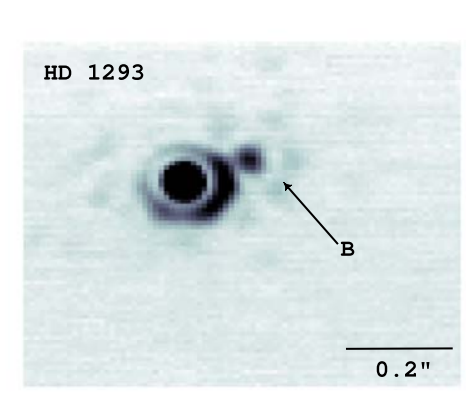

HD 1388

HD 4406
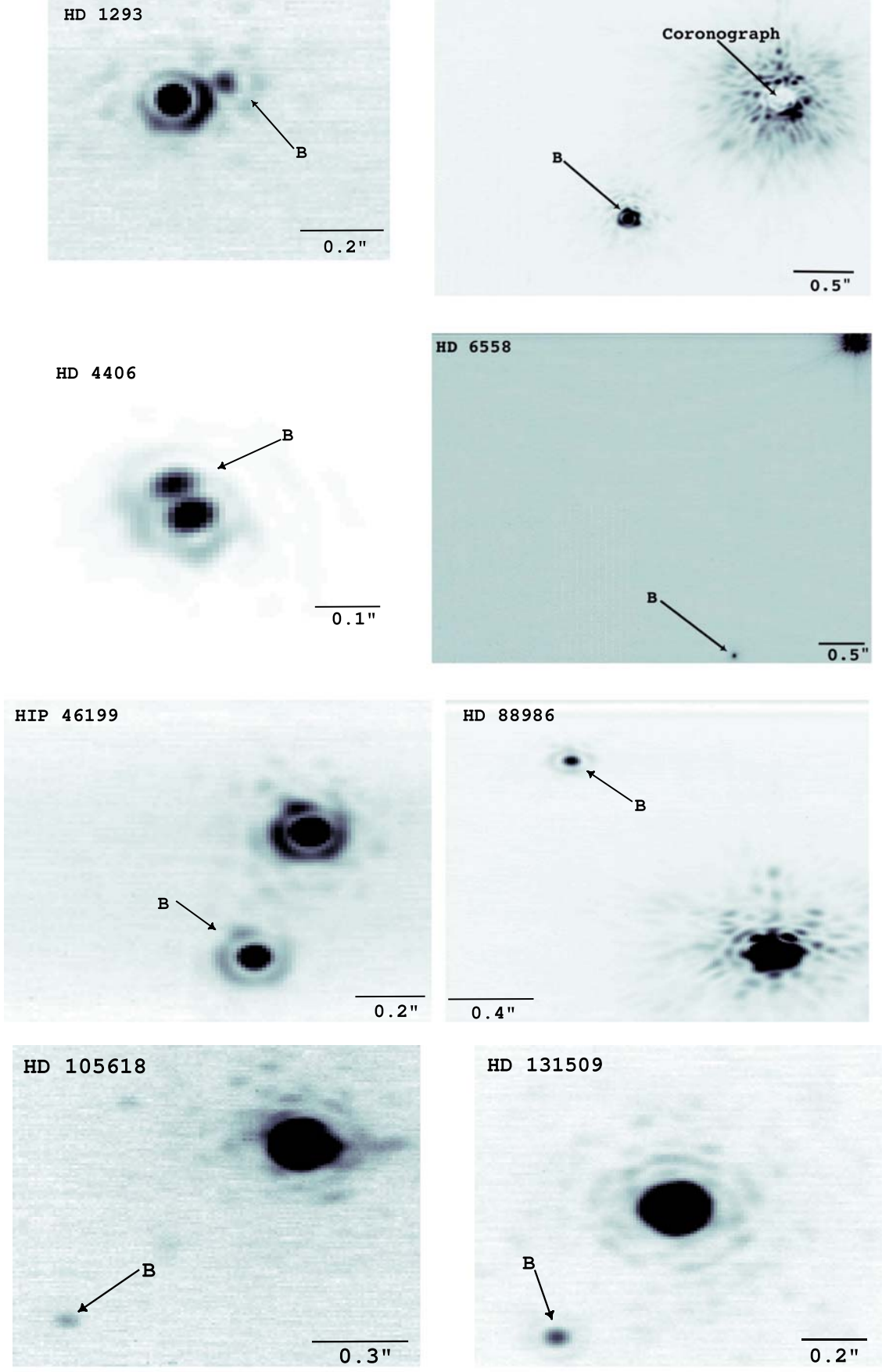

$.4^{11}$
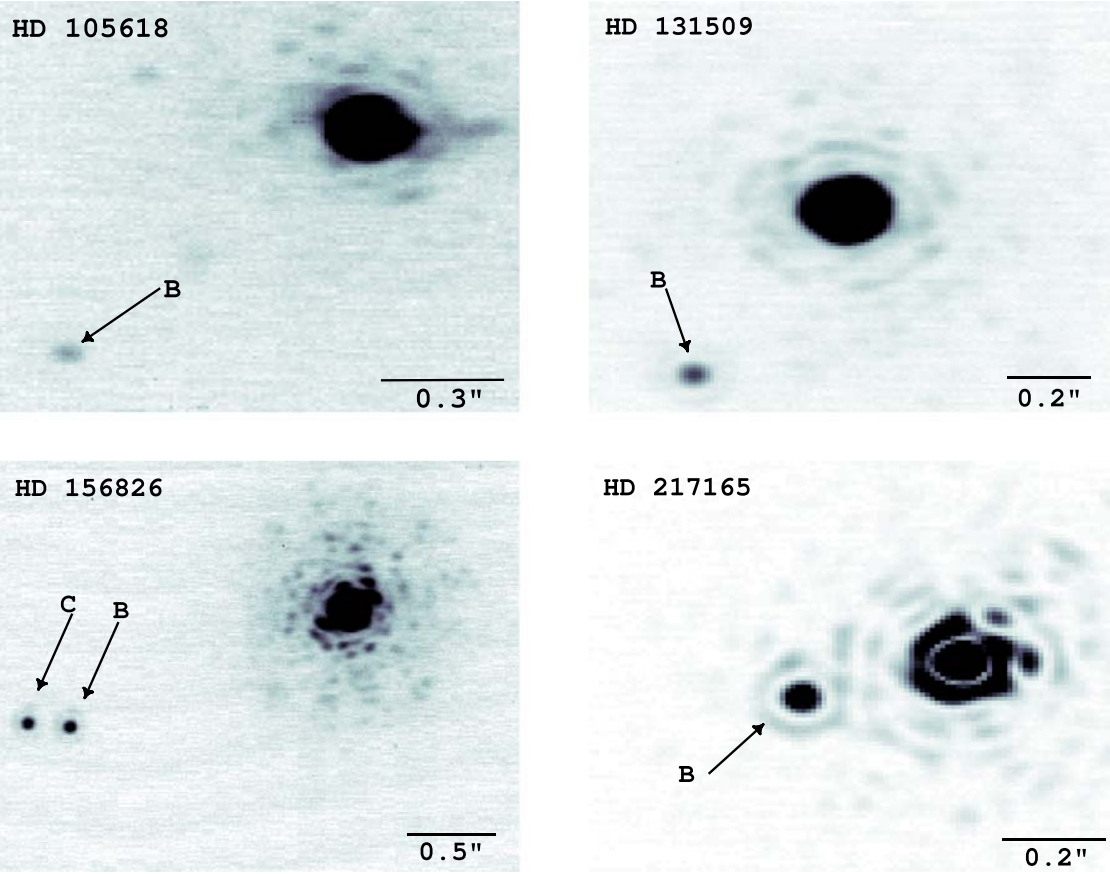

HD 217165

Figure 10. High-resolution AO images of candidate companions. 


\section{A.5. Common Proper Motion Plots of Confirmed Companions}

Figure 11 displays the space motion of each confirmed companion and that of a background object at infinity as indicated by the tracks plotted in teal. Proper motions in the north and east directions falling off the teal track indicate that the companion is comoving and not a background object.
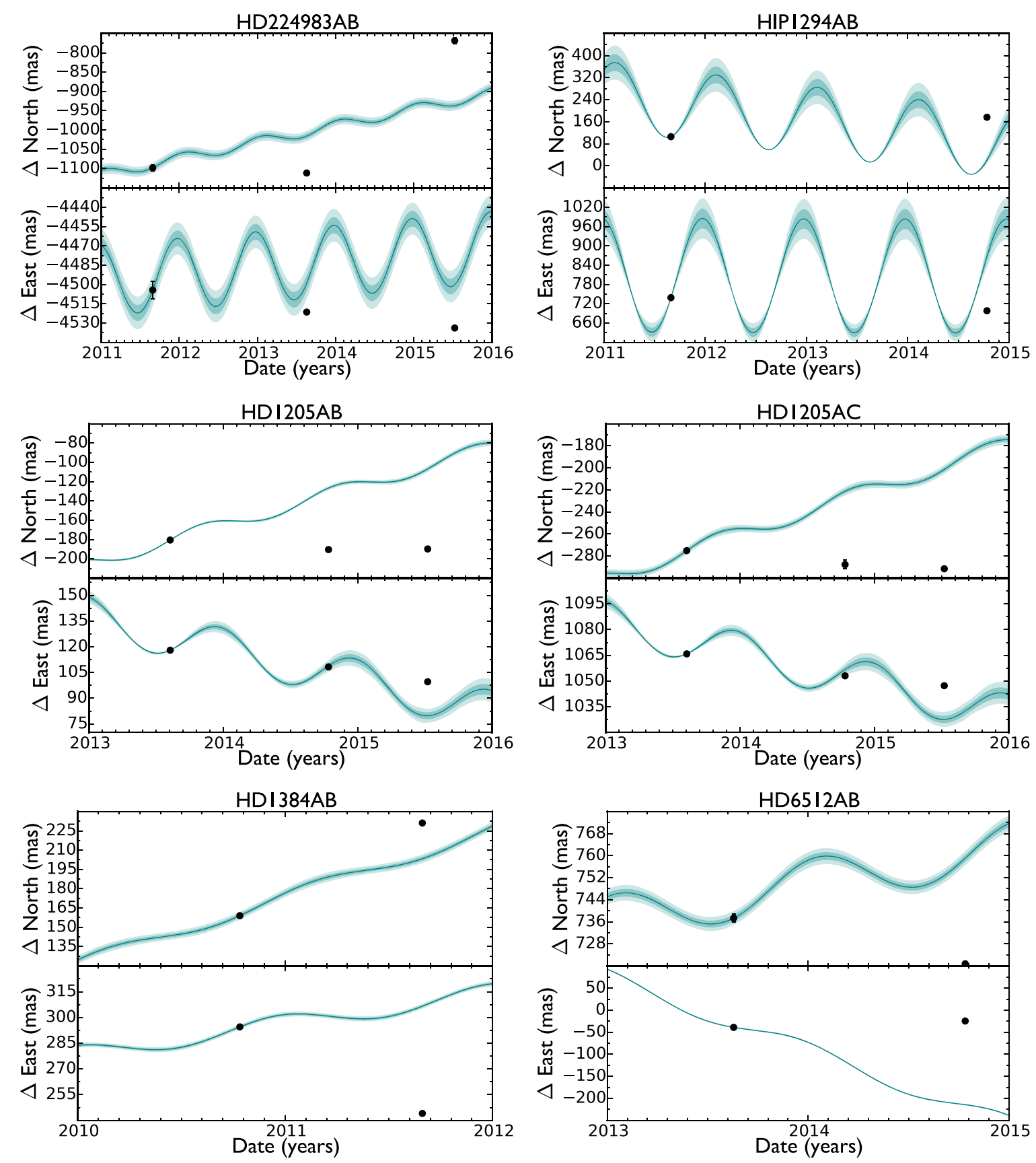

Figure 11. Confirmation of co-movement of companion: the curved lines are the path taken of an infinitely distant background object. Plotted points are the astrometric measurements of the companion. Given that the measurements do not fall along the path of a background object, the companion shares the same space motion and is gravitationally associated. 

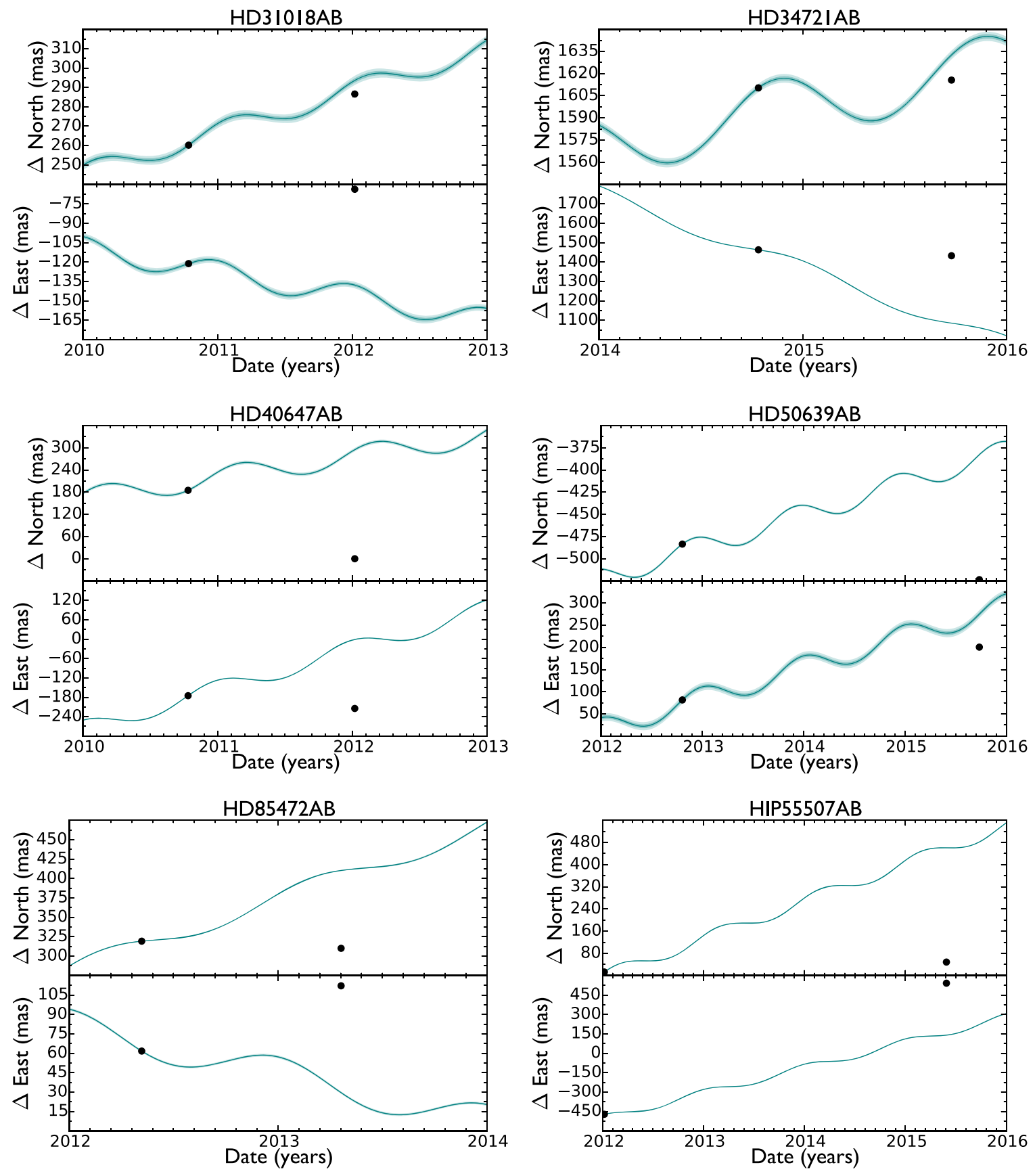

Figure 11. (Continued.) 

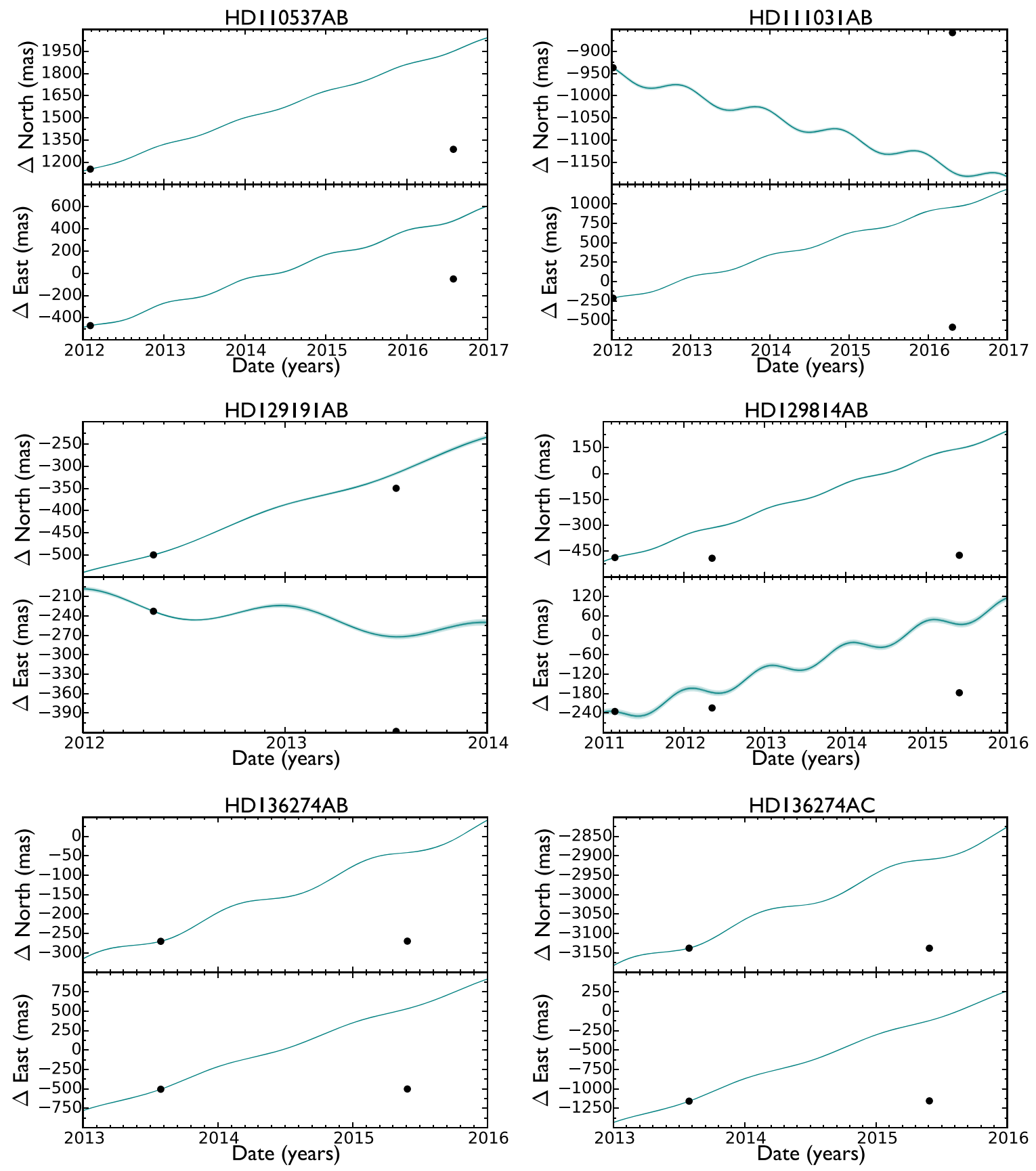

Figure 11. (Continued.) 

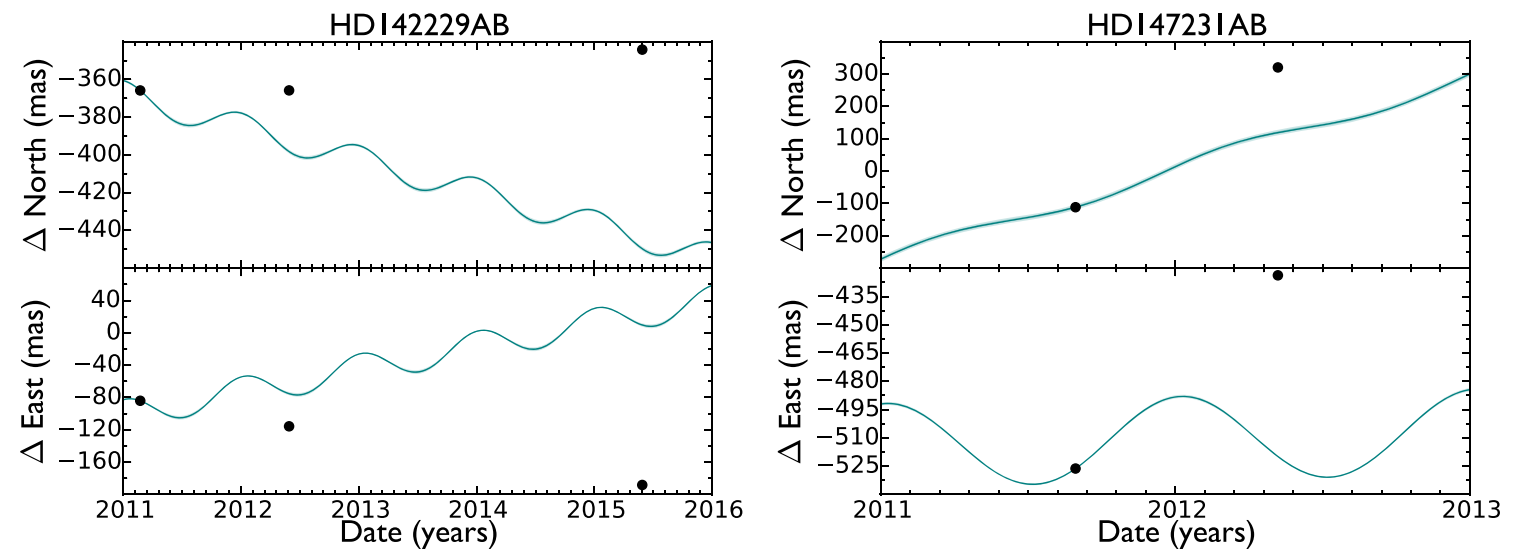

HDI554I3AB
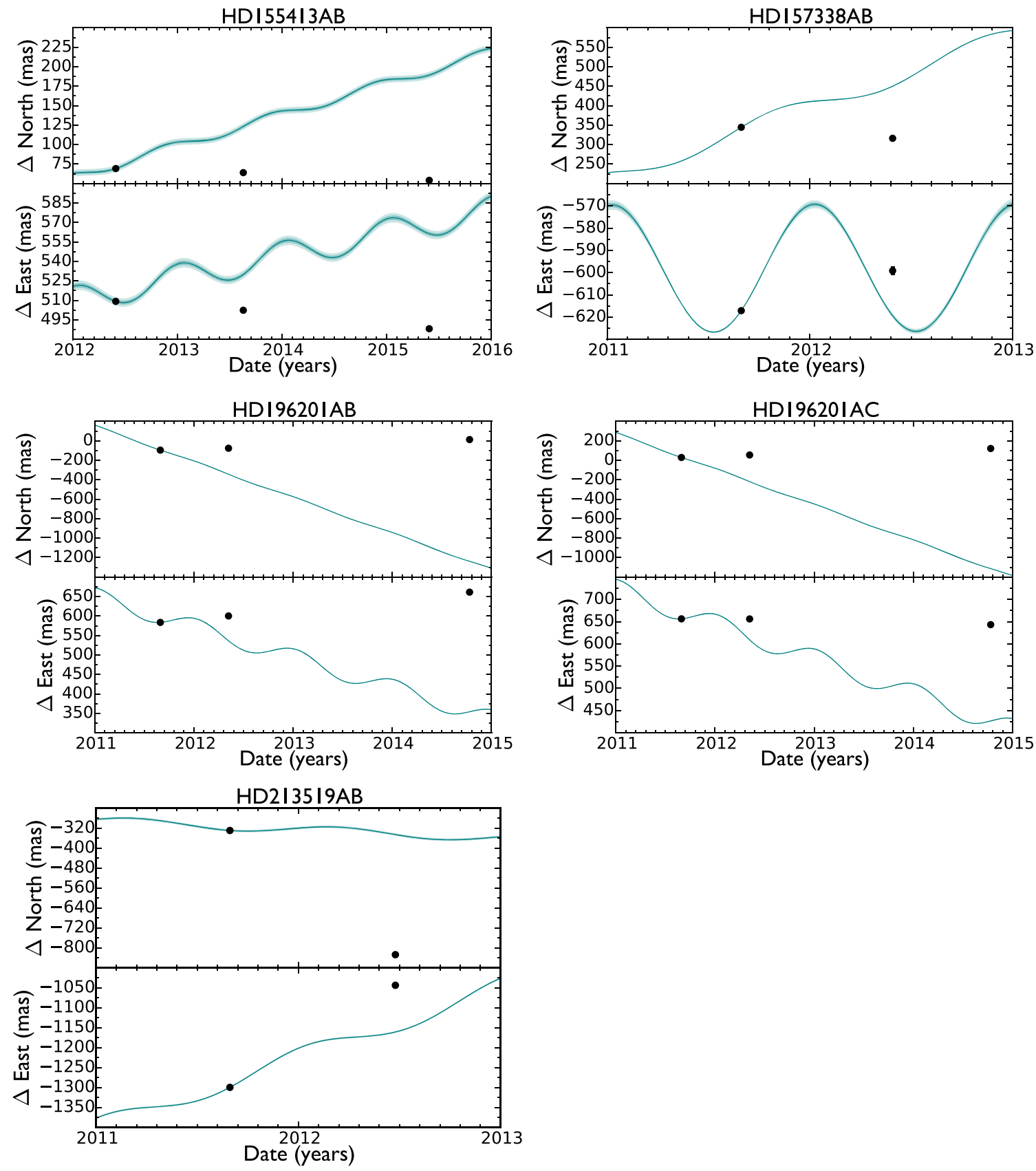

Figure 11. (Continued.) 


\section{ORCID iDs}

Erica J. Gonzales (i) https://orcid.org/0000-0002-9329-2190

Justin R. Crepp (1) https://orcid.org/0000-0003-0800-0593

Eric B. Bechter (1) https://orcid.org/0000-0001-8725-8730

Charlotte M. Wood (i) https://orcid.org/0000-0003-4773-4602

Benjamin T. Montet (1) https://orcid.org/0000-0001-

7516-8308

Howard Isaacson (16) https://orcid.org/0000-0002-0531-1073

Andrew W. Howard (1) https://orcid.org/0000-0001-

8638-0320

\section{References}

Allard, F., Hauschildt, P. H., Alexander, D. R., Tamanai, A., \& Schweitzer, A. 2001, ApJ, 556, 357

Allard, F., Homeier, D., Freytag, B., Schaffenberger, W., \& Rajpurohit, A. S. 2013, MSAIS, 24, 128

Baraffe, I., Homeier, D., Allard, F., \& Chabrier, G. 2015, A\&A, 577, A42

Bechter, E. B., Crepp, J. R., Ngo, H., et al. 2014, ApJ, 788, 2

Biller, B. A., Vos, J., Bonavita, M., et al. 2015, ApJL, 813, L23

Bowler, B. P., Dupuy, T. J., Endl, M., et al. 2018, AJ, 155, 159

Boyajian, T. S., von Braun, K., van Belle, G., et al. 2012, ApJ, 757, 112

Burrows, A. 2001, arXiv:astro-ph/0011132

Butler, R. P., Vogt, S. S., Laughlin, G., et al. 2017, AJ, 153, 208

Chabrier, G., Baraffe, I., Allard, F., \& Hauschildt, P. 2000, ApJ, 542, 464

Cheetham, A., Bonnefoy, M., Desidera, S., et al. 2018, A\&A, 615, A160

Crepp, J. R., Gonzales, E. J., Bechter, E. B., et al. 2016, ApJ, 831, 136

Crepp, J. R., Johnson, J. A., Fischer, D. A., et al. 2012a, ApJ, 751, 97

Crepp, J. R., Johnson, J. A., Howard, A. W., et al. 2012b, ApJ, 761, 39

Crepp, J. R., Johnson, J. A., Howard, A. W., et al. 2013a, ApJ, 771, 46

Crepp, J. R., Johnson, J. A., Howard, A. W., et al. 2013b, ApJ, 774, 1

Crepp, J. R., Johnson, J. A., Howard, A. W., et al. 2014, ApJ, 781, 29

Crepp, J. R., Principe, D. A., Wolff, S., et al. 2018, ApJ, 853, 192

Cutri, R. M., Skrutskie, M. F., van Dyk, S., et al. 2003, yCat, 2246

Dupuy, T. J., \& Liu, M. C. 2012, ApJS, 201, 19

Dupuy, T. J., \& Liu, M. C. 2017, ApJS, 231, 15

Dupuy, T. J., Liu, M. C., Leggett, S. K., et al. 2015, ApJ, 805, 56
Faherty, J. K., Riedel, A. R., Cruz, K. L., et al. 2016, ApJS, 225, 10

Foreman-Mackey, D., Hogg, D. W., Lang, D., \& Goodman, J. 2013, PASP, 125,306

Gaia Collaboration, Brown, A. G. A., Vallenari, A., et al. 2018, A\&A, 616, A1 Giguere, M. J., Fischer, D. A., Howard, A. W., et al. 2012, ApJ, 744, 4

Goodman, J., \& Weare, J. 2010, Commun. Appl. Math. Comput. Sci., 5, 65

Hayward, T. L., Brandl, B., Pirger, B., et al. 2001, PASP, 113, 105

Høg, E., Fabricius, C., Makarov, V. V., et al. 2000, A\&A, 355, L27

Howard, A. W., Johnson, J. A., Marcy, G. W., et al. 2010, ApJ, 721, 1467

Jenkins, J. S., Jones, H. R. A., Pavlenko, Y., et al. 2008, A\&A, 485, 571

Kirkpatrick, J. D., Gelino, C. R., Cushing, M. C., et al. 2012, ApJ, 753, 156

Koen, C., Kilkenny, D., van Wyk, F., \& Marang, F. 2010, MNRAS, 403, 1949 Konopacky, Q. M. 2013, MmSAI, 84, 1005

Lada, C. J., \& Lada, E. A. 2003, ARA\&A, 41, 57

Lindegren, L., Lammers, U., Bastian, U., et al. 2016, A\&A, 595, A4

Liu, M. C., Dupuy, T. J., \& Ireland, M. J. 2008, ApJ, 689, 436

Liu, M. C., Fischer, D. A., Graham, J. R., et al. 2002, ApJ, 571, 519

Mann, A. W., Dupuy, T., Muirhead, P. S., et al. 2017, AJ, 153, 267

Marcy, G. W., \& Butler, R. P. 1992, PASP, 104, 270

Marcy, G. W., Butler, R. P., \& Fischer, D. A. 1999, in ASP Conf. Ser. 185 IAU Coll. 170: Precise Stellar Radial Velocities, Doppler Detection of Extra-Solar Planets, ed. J. B. Hearnshaw \& C. D. Scarfe (San Francisco, CA: ASP), 121

Montet, B. T., Crepp, J. R., Johnson, J. A., Howard, A. W., \& Marcy, G. W. 2014, ApJ, 781, 28

Muirhead, P. S., Dressing, C. D., Mann, A. W., et al. 2018, AJ, 155, 180

Ngo, H., Knutson, H. A., Bryan, M. L., et al. 2017, AJ, 153, 242

Oja, T. 1996, yCat, 2182

Pecaut, M. J., \& Mamajek, E. E. 2013, ApJS, 208, 9

Ricker, G. R., Winn, J. N., Vanderspek, R., et al. 2015, JATIS, 1, 014003

Saumon, D., \& Marley, M. S. 2008, ApJ, 689, 1327

Service, M., Lu, J. R., Campbell, R., et al. 2016, PASP, 128, 095004

Skrutskie, M. F., Cutri, R. M., Stiening, R., et al. 2006, AJ, 131, 1163

Torres, G. 1999, PASP, 111, 169

Valenti, J. A., \& Fischer, D. A. 2005, ApJS, 159, 141

Vogt, S. S., Allen, S. L., Bigelow, B. C., et al. 1994, Proc. SPIE, 2198 362

Wizinowich, P., Acton, D. S., Shelton, C., et al. 2000, PASP, 112, 315

Wood, C. M., Boyajian, T., von Braun, K., et al. 2019, ApJ, 873, 83

Yelda, S., Lu, J. R., Ghez, A. M., et al. 2010, ApJ, 725, 331 